\title{
The analytical method in soil mechanics
}

\author{
R. E. GIBSON, DSc(Eng), FICE*
}

I should like, first, to thank the British Geotechnical Society for inviting me to deliver the Fourteenth Rankine Lecture, and then to thank Professor Nash not only for his kind introductory remarks but also for his generous support and for the relative freedom I have enjoyed during my time at King's College. It is also a pleasure to have this opportunity to acknowledge the help and encouragement I received from Professor Skempton while I was at Imperial College -and, indeed, this was most generously given even when I was occupied elsewhere. To these names I should like to add that of Dr Cooling who allowed me while at the Building Research Station to learn some mathematics from Dr McNamee; and who also required me to engage in field work between January and March.

\section{INTRODUCTION}

I have entitled this Lecture 'The analytical method in soil mechanics' and this raises the question of what meaning is to be given to the word 'analytical'. The Oxford English Dictionary defines analysis as 'the resolution of anything complex into its simple elements', but in the seventeenth century a special meaning arose in the field of mathematics, which the dictionary renders as 'the resolving of problems by reducing them to equations'.

Resolution into simple elements seems to be instinctive and part of our everyday experience, but it is rarely a discrete mental activity, and is attended invariably by synthesis as we proceed from insight to consequences. Deliberate analysis, however, to be effective, must have regard to the conscious abstraction from a situation of those elements which seem likely to have significance in the context of our objectives. The elevation at which London Clay changes in colour from brown to blue might, and very often would, be quite inconsequential; but not, perhaps, if we were concerned with assessing the depth of open joints and fissures.

When what seems irrelevant has been discarded-and clearly this selection requires judgement and experience-a synthesis is needed which allows the remaining factors to be interrelated and structured. This synthesis can often be achieved by means of a model, but to be of use it will have to be one we can handle with the means at our disposal and be able to make reasonable qualitative or quantitative predictions without a disproportionate amount of effort. These conditions are likely to be met, especially in the case of a mathematical model, only if simplifications are introduced by idealizing to some extent the real situation. The choice of what simplifications to make will be guided by our prior expectation of the likely effect of each on the predictions. It may be good tactics and sometimes the only feasible course open, to oversimplify the model and then study seriatim the influence of the discarded factors. A sensitivity study of this kind, which enlarges the notion of a parametric study, can deepen insight and sometimes wholly unexpected results emerge.

Having arrived at a tentative model by analysing, abstracting, synthesizing and idealizing, we come to the second sense of the word analysis. The definition is inadequate, for although describing the behaviour of the model in mathematical terms is a necessary first step-and this

- Professor of Engineering Science, King's College, University of London. 
will usually entail setting up governing equations--problems are not resolved by this procedure, for questions are neither answered nor predictions made. To achieve this requires the equations to be solved, account being taken of constraints, such as boundary conditions, appropriate to any problem.

If to the model we address questions of a general character, without reference to any particular problem, we may be fortunate and discover results of great generality: the principle of superposition of linear systems is an example. Sometimes no unique answer can be given; this is a feature of models and problems which involve instability of one sort or another.

A perfect, uniform, axially loaded strut in a uniform environment will not bow when the load is increased; an idealized approach has removed the possibility of this type of deformation, but this prediction is wholly at variance with experience.

How will an oil tank, founded on a clay stratum, tilt and settle when loaded? At loads well below the failure load the assumption that the clay is uniform may lead to predictions which conform with experience. At higher loads, however, small non-uniformities in the deformation and strength characteristics of the clay will play an increasingly important role. The stress-strain curve for the clay need show only a small degree of work-softening beyond the peak for the prediction of uniform settlement to be wholly unreliable. In this case the influence of slight non-uniformity is greatly increased by the presence of instability due to worksoftening.

The analytical method, therefore, sometimes forces us to acknowledge that some apparently well-posed problems do not possess a unique solution. Nevertheless, the influence of various assumed patterns of non-uniformity on the tank settlement can be examined, and much may be learnt from these sensitivity studies.

Fortunately, neither the immediate settlement characteristics at low working loads, nor the maximum load-carrying capacity of the clay are sensitive to small non-uniformities, although the average properties do not always dictate the response. Some problems of this type where cause and effect are not disproportionately related, will be considered.

\section{IMMEDIATE SETTLEMENTS}

\section{The influence of heterogeneity and anisotropy}

The first example concerns the application of elastic theory to predict the immediate settlement of a structure founded on a layer of saturated clay. This choice was not dictated by the engineering importance or otherwise of the problem, but because it reveals the role appropriate to analysis. The use of an elastic model for clay at low working stresses has received much attention and the limitations and assumptions involved are well known. ${ }^{1}$

Lamé and Clapeyron (1833) had developed by 1828 formulae governing the deformations of a homogeneous and isotropic clastic half-space loaded normal to its planc boundary. Their formulae were so complex that Lamé (1852) in his lectures twenty years later confessed that they were too complicated to be applied in practice. However, Boussinesq (1885) using a potential function method, succeeded in developing the solution in a form which could be used directly (Mayer, 1954). The publication by Love (1928) of the paper 'The stresses produced in a semi-infinite solid by pressures acting on part of the boundary' marks, roughly, the end of a period during which mathematicians were studying these problems for their intrinsic interest. At about the same time the recognition by engineers that these solutions were relevant to their own needs, began a new period of activity which continues to the present.

\footnotetext{
${ }^{1}$ See, for example, Davis and Poulos (1963, 1968); Lambe (1964); Kérisel and Quatre (1966, 1968); Pérez-La Salvia
} et al. (1966); Moore and Spencer (1969); Wroth (1971); Henkel (1971); Simons (1971); Poulos and Davis (1974). 
One principle of great importance to engineers emerged at an early stage and was certainly known to Boussinesq: that for linear problems of elasticity the principle of superposition holds. The solution to problems of combined surface loading can be obtained by adding together solutions to problems involving simple surface loading; in particular, the solution to those problems where vertical surface loading is prescribed can be derived from a knowledge of the stresses and displacements induced by a single point load. This is the basis of the well-known Newmark charts (Newmark, 1942, 1947) and the Poulos sector method (Poulos, 1967).

So far as I have been able to discover all the earlier studies except one (Michell, 1900) assumed the half-space or layer to be uniform in elastic properties (i.e. homogeneous) and to possess the same properties in all directions (i.e. isotropic). Real soil strata invariably increase in rigidity with depth and this reflects the increasing overburden pressure. Furthermore, laboratory tests on samples of clay secured with the minimum of disturbance from depth, indicate that their deformation properties often differ in the vertical and horizontal directions. This anisotropy is thought to arise chiefly from the difference between the vertical and horizontal effective stresses which develop and increase during deposition, and which in turn leads to some preferred orientation of the plate-shaped clay particles; and partly to the presence of thin horizontal seams of coarser-grained soil which results from the varying conditions during the period of deposition of the sediment.

The following question now arises: to what extent will the predictions of elastic theory be modified if heterogeneity and anisotropy are taken into account in the model? The importance of anisotropy has, of course, been appreciated for some time (Crawford and Burn, 1962; Hanna, 1965; Duncan and Seed, 1966; Mitchell 1972; Atkinson, 1973) but the influence of heterogeneity has been less widely recognized (Burland et al., 1973). There is no way of answering this question other than by examining particular cases. The solution to one problem may suffice to indicate a general trend, but only if it is selected with care. A single vertical point load, unfortunately, gives rise to a singularity in the stress field at its point of application, which leads to an infinite settlement there: a simplification which might be judged desirable on mathematical grounds presents difficulties of another kind. Uniform loading over a strip of the surface certainly restricts the deformation so that the problem becomes two-dimensional and therefore simpler, but from this solution the case of arbitrary surface loading cannot be derived using super-position. However, a load distributed uniformly over a circular area still presents essentially a two-dimensional situation, and from it non-uniform loading over areas of any shape can be built up using superposition of solutions which principle is still valid even in the presence of those types of heterogeneity and anisotropy which we consider here.

The heterogeneous problem was suggested to me some years ago by Dr Noel Simons of Surrey University, but I was unaware then that certain special cases had been considered by Griffith (1929), Fröhlich (1934), Holl (1940) and by Borowicka (1943) as well as by a number of Russian workers (Mikhlin, 1935; Klein, 1956; Koronev, 1957; Sherman, 1958; Popov, 1959; Lekhnitskii, 1962; Zaretsky and Tsytovich, 1965).

Without the added complication of anisotropy there were grounds for hoping that a complete solution by algebraic means would be possible. For simplicity it was assumed that the clay stratum was infinitely deep and incompressible - to model the immediate response of saturated clays subject to sudden loading. There was a good deal of evidence (for London Clay see Skempton and Henkel, 1957; Ward, et al., 1959, 1965) that the undrained Young's modulus $E$ increases approximately linearly with depth $z$ according to the relation

$$
E(z)=E(0)+\lambda z
$$




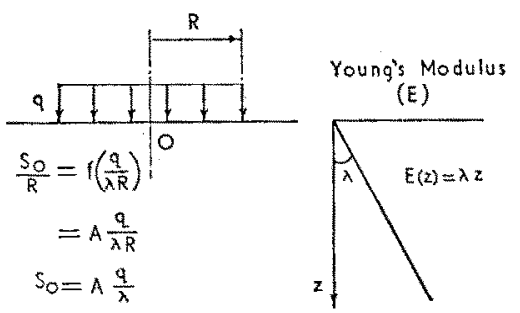

Fig. 1
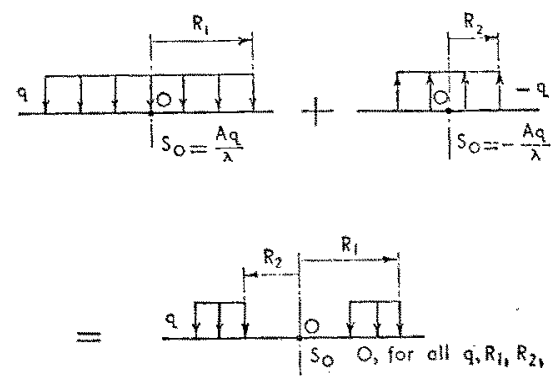

Fig. 2

Two extreme cases can be identified
(a) $\lambda=0$
(b) $E($ o $)=0$.

The first of these is the classical case and would provide a useful check of the general analysis. The second is unrealistic in one important respect because immediately beneath the load the undrained modulus and therefore the undrained strength of the clay would be zero. It was difficult to see how the clay could avoid failing there, in which case the simple elastic model would, at least locally, become inadequate. For reasons which will appear shortly it turns out that this objection is not quite so serious as it might appear.

A solution to the problem has been published (Gibson, 1967). The most important result found, which is connected with the extreme case when the surface modulus $E(o)$ is zero, can be reached using simple reasoning and without recourse to mathematics.

Consider a pressure $q$ uniformly distributed over a circular area of radius $R$ at the surface of an elastic half-space, the Young's modulus of which increases, from zero at the surface, linearly with depth (Fig. 1).

When the surface modulus is zero, the only quantities involved in the problem are the pressure $q$, the radius of the loaded area $R$ and $\lambda$ the rate of increase of the undrained modulus with depth. We seek, first, to relate the settlement $S_{0}$ at the centre of the circular loaded area, to these three quantities. The Poisson's ratio $\nu$ of the medium is assumed to be uniform and, in the special case of undrained deformation, $y=\frac{1}{2}$.

The four magnitudes $q, R, \lambda$ and $S_{0}$ involve dimensions of stress and length alone, and from these, two dimensionless groups can be formed, namely $S_{0} / R$ and $q / \lambda R$. By Riebouchinski's theorem (Buckingham, 1921) or by employing reasoning encountered in elementary fluid mechanics, these dimensionless groups must be connected functionally by a relation of the form

$$
S_{\mathrm{o}} / R=\mathrm{f}(q / \lambda R)
$$

As the material is linear in its response, a doubling of the pressure $q$ will lead to a doubling of the settlement $S_{0}$. Furthermore, when the pressure is zero the settlement is also zero. The only functional form which meets these conditions is a linear relation

$$
S_{\mathrm{o}} / R=A(q / \lambda R)
$$

where $A$ is some constant which depends only on Poisson's ratio. The centre settlement is therefore

$$
S_{0}=A q / \lambda
$$

which does not involve the radius $R$ of the loaded area. This result, so far, is restricted to the particular loading considered and to the settlement at the centre.

As neither Young's modulus nor Poisson's ratio varies in a horizontal direction it can be 


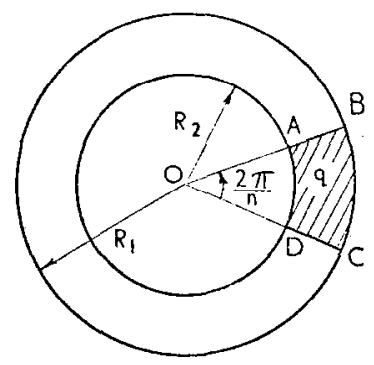

Fig. 3

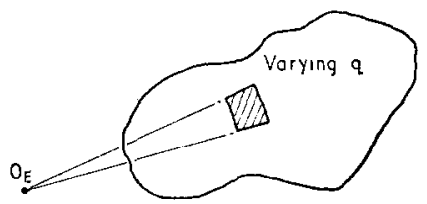

Fig. 4

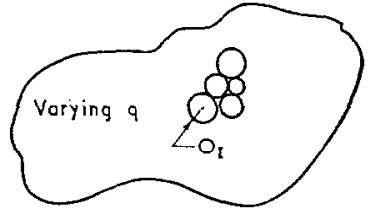

Fig. 5

shown that superposition is valid for additions of surface loading and we attempt now to generalize the result by appeal to this principle.

The settlement at the centre $\mathrm{O}$ of a circular uniformly loaded area of radius $R_{1}$ (Fig. 2) will be

$$
S_{\mathrm{o}}=A q / \lambda
$$

and at the centre of a concentric circular area of radius $R_{2}<R_{1}$ to which a uniform tension $-q$ is applied, it will be $-A q / \lambda$. Combining these two solutions establishes that a pressure $q$ applied over an annulus extending from $R_{2}$ to $R_{1}$ will lead to no settlement at the centre point $\mathrm{O}$, whatever the values of $R_{1}$ and $R_{2}$.

Divide the annulus into $n$ sectors each subtending an angle $(2 \pi / n)$ at the centre (Fig. 3 ). If the settlement at $O$ due to a pressure $q$ acting on a single sector is $\Delta S_{\circ}$ then, using superposition again, the settlement at the centre will be $n \Delta S_{0}$ due to the pressure acting over the whole annulus, since the contribution of each annular sector is the same. Therefore

$$
S_{\mathrm{o}}=n \Delta S_{\mathrm{o}} \quad . \quad . \quad . \quad . \quad . \quad . \quad . \quad . \quad . \quad .
$$

But as $S_{0}=0$, it follows that $\Delta S_{0}=0$ and this result is valid irrespective of the dimensions or radial distance of the area $A B C D$ from the point $O$.

This argument may be extended to determine the surface settlement of the medium when subjected to a non-uniform vertical pressure distributed over an area of the surface of arbitrary shape. Consider a point $\mathrm{O}_{\mathrm{E}}$ (Fig. 4) exterior to the loaded area and divide, by means of arcs centre $O_{E}$ and radii through $O_{E}$, the area into a sufficiently large number of elementary areas to ensure that, to any desired closeness of approximation, the pressure on every small area is uniform. By the result already established, the settlement at $\mathrm{O}_{\mathrm{E}}$ due to the pressure on any of these elementary areas is zero and therefore the settlement at $\mathrm{O}_{\mathrm{E}}$ due to the whole load is zero. This result is true for all points exterior to the load.

Divide the loaded area into a sufficiently large number $N$ of infinitesimal circular areas to occupy the whole area (Fig. 5). Consider any point $\mathrm{O}_{\mathrm{I}}$ interior to the area which is also the centre of one of the circles. The settlement at $\mathrm{O}_{\mathrm{I}}$ due to the local pressure $q_{\mathrm{L}}$ is $A q_{\mathrm{L}} / \lambda$, but since the point $\mathrm{O}_{\mathrm{I}}$ is cxtcrior to the remainder of the load this does not contribute to the settlement. Therefore, at any point within the loaded area the settlement is $A q_{\mathrm{L}} / \lambda$.

This analysis shows that the medium will settle under vertical pressure in the same manner as a uniform bed of springs. This simple spring model is the basis of the ideas underlying the method of subgrade reaction (Terzaghi, 1955) which has allowed structural engineers for many years to estimate bending moments and shear forces in foundation slabs and rafts. But the above analysis allows the discrete springs to be replaced by an elastic continuum capable of transmitting both shear and horizontal stress. Evidently, the coefficient of subgrade reaction depends directly on $\lambda$, but to establish the full connexion the unknown constant $A$ must be found. 

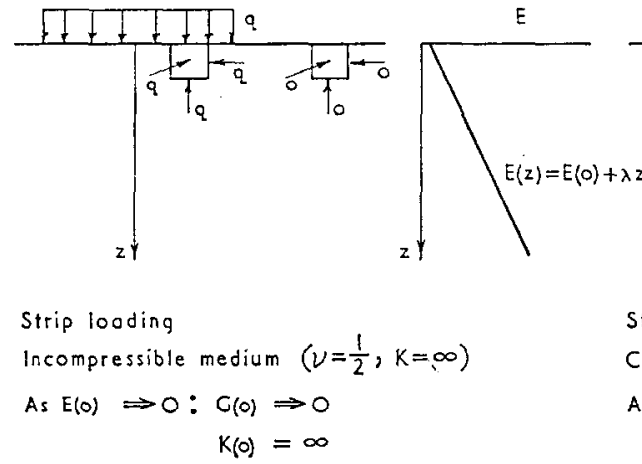

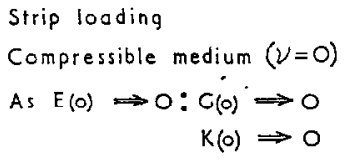

For this a complete analysis is required and it has been shown (Gibson, 1967) that, for an incompressible medium, $A=3 / 2$. If the medium is compressible-that is, if Poisson's ratio is less than one half the constant $A$ becomes infinite (Awojobi and Gibson, 1973). Why should this be so?

An examination of the stress distribution shows that immediately beneath a strip load, for example (Fig. 6), the three principal stresses are equal to $q$ if, and only if, the medium is incompressible. In this case a thin layer of incompressible 'fluid' exists at the surface, and this is under an isotropic state of stress. Neither volumetric nor distortional strains can develop and this thin layer does not therefore contribute to the settlement. ${ }^{2}$ However, if Poisson's ratio is less than one half, both the shear modulus and the bulk modulus of this thin 'skin' are zero as Young's modulus there is zero. Furthermore, the principal stresses in this case are found to be unequal (Gibson and Sills, 1971) and this allows infinite volumetric and shear strains to develop locally. Whether or not an infinite settlement occurs depends on which influence predominates: the magnitude of the strains, or the thinness of the layer. A mathematical argument to settle this point (Awojobi and Gibson, 1973) shows that the infinite strains do, in general, lead to surface settlements which are logarithmically infinite.

Unfortunately, therefore, the simple result that the surface settlement is proportional to the local intensity of pressure holds only if the medium is incompressible.

However, the general case when the surface value $E(0)$ of Young's modulus is not zero has been solved for arbitrary values of Poisson's ratio (Awojobi and Gibson, 1973) and the surface settlements evaluated (Brown and Gibson, 1972, 1973); more useful, although less simple, results are obtained. The variation in the shape of the profiles of surface settlement with the parameter $E(\mathrm{o}) / \lambda R$, which is a convenient measure of the degree of heterogeneity, is shown in Fig. 7 for an incompressible half-space. As this parameter decreases the heterogeneity becomes more pronounced, the settlement becomes more uniform except close to the edge of the load. For other values of Poisson's ratio results which can hardly be distinguished from these were obtained. The influence of Poisson's ratio has almost been removed by plotting the ratio of the settlement $S(r)$ to the centre settlement $S_{0}$ and in the homogeneous case $(\lambda=0)$ this result is exact. Although both $S(r)$ and $S_{\mathrm{o}}$ are infinite when the surface modulus is zero (except when the medium is incompressible) their ratio is either unity or zero.

This analysis indicates that the shape of the deformed surface is likely to be very sensitive to the stiffening of the soil with depth. As almost all clays show this tendency, save for the desiccated upper zone of normally consolidated clays, it is to be expected that a uniform surface

2 This is strictly a double-limit problem. The order in which the operations $\nu \rightarrow \frac{1}{2}$ and $E(0) \rightarrow 0$ are applied apparently affects the surface value of the bulk modulus $K(0)=E(0) / 3(1-2 \nu)$ but not the surface shear modulus $G(0)=E(0) / 2(1+\nu)$ at the double limit. It has been shown, however (Awojobi and Gibson, 1973) that the same surface settlement is obtained independent of the path by which the limit point is approached. 


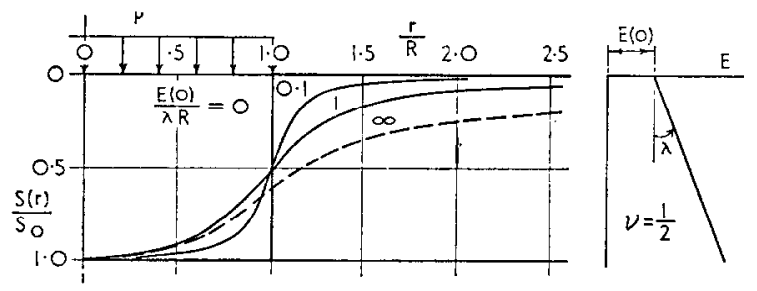

Fig. 7. Surface settlement profile of linearly heterogeneous incompressible elastic half-space due to uniformly loaded circular area

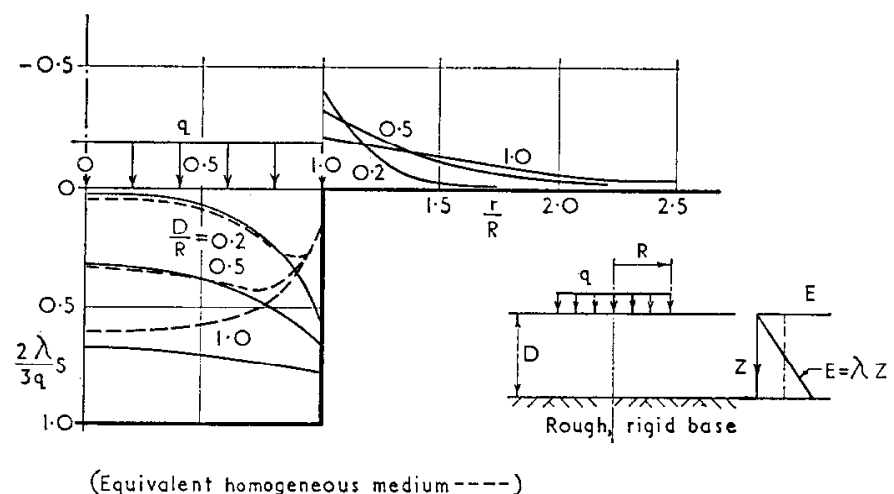

Fig. 8. Surface settlement profiles of heterogeneous and equivalent homogeneous incompressible elastic layer due to uniformly loaded circular area.

pressure would lead to a more uniform settlement profile than is predicted by simple elastic theory.

Unlike the settlement, the stress distribution is found to be comparatively insensitive, except the lateral stresses which are sensitive at small values of $\nu$ (Gibson and Sills, 1971; Carrier and Christian, 1973) to the heterogeneity and, indeed, it becomes progressively less sensitive the closer Poisson's ratio approaches one half. When, for example, the surface loading is along an infinite strip the stresses when $E(o)=0$ are exactly equal to those which arise in the classical uniform medium (Lekhnitskil, 1962; Gibson, 1967).

These preliminary studies were based on the assumption that the medium is infinitely deep and this was done chiefly to avoid mathematical difficulties. It is well known that the layer depth has an important influence on the settlement profile in the homogeneous case (Steinbrenner, 1934) and that reducing the layer thickness tends to make the settlement more uniform. When the layer is incompressible and the base is rough this is accompanied by some surface heave outside the loaded area. A study of the incompressible layer (Gibson et al., 1971) with a zero surface modulus and a base which adheres completely to the underlying rigid material showed that as the layer became progressively thinner the settlement became less uniform. However, this non-uniformity is found to be confined to the edge of the loaded area where the soft surface layers are squeezed laterally outwards and upwards to form a lip (Fig. 8). Further studies of the layer case have been carried out using numerical methods by my colleague Mr Andrews and by Carrier and Christian at MIT. They were easily able to tackle the more realistic case of a finite surface modulus and this, together with the added difficulty of a finite layer depth, renders a numerical approach much more attractive. ${ }^{3}$

\footnotetext{
${ }^{3}$ Numerical methods abhor zeros and infinities, but their presence can sinplify the analyst's task.
} 
Finally, it was decided to return to the half-space problem and investigate heterogeneity coupled with anisotropy. Very extensive studies of the anisotropic, homogeneous half-space have been published recently by Gerrard and his colleagues (Gerrard and Harrison, 1970, 1971) and by others (Milovic and Tuzot, 1970; Milovic, 1972; Nayak, 1973). The simplest case, and the one likely to be encountered in geotechnics, occurs when the principal axes of anisotropy are everywhere vertical and horizontal and when the properties in all horizontal directions are the same. The basic modification to the earlier work demanded by this generalization concerns the stress-strain relations, for instead of two independent elastic constants $E$ and $\nu$, five are now involved: two Young's moduli $E_{\mathrm{V}}$ and $E_{\mathrm{H}}$ a shear modulus $G_{\mathrm{VH}}$ and two Poisson's ratios $\nu_{\mathrm{HV}}$ and $\nu_{\mathrm{HH}}$ (Hearmon, 1961; Lekhnitskiǐ, 1963; Pickering, 1970). Taking the axis $y$ in the vertical direction and axes $x$ and $z$ in the plane of the surface, these take the form

$$
\begin{aligned}
& e_{x}=\frac{\sigma_{x}}{E_{\mathrm{II}}}-\nu_{\mathrm{HV}} \frac{\sigma_{y}}{E_{\mathrm{H}}} \nu_{\mathrm{HH}}-\frac{\sigma_{z}}{E_{\mathrm{H}}} \quad \cdot \quad \cdot \quad \cdot \quad \cdot \quad \cdot \quad . \quad \cdot \\
& e_{y}=-\nu_{\mathrm{HV}} \frac{\sigma_{x}}{E_{\mathrm{H}}}+\frac{\sigma_{y}}{E_{\mathrm{V}}}-\nu_{\mathrm{HV}} \frac{\sigma_{z}}{E_{\mathrm{H}}} \text {. . . . . . . . . . } \\
& e_{z}=-\nu_{\mathrm{HH}} \frac{\sigma_{\chi}}{E_{\mathrm{H}}}-\nu_{\mathrm{HV}} \frac{\sigma_{y}}{E_{\mathrm{H}}}+\frac{\sigma_{z}}{E_{\mathrm{H}}} \text {. . . . . . . . . . } \\
& \gamma_{x y}=\tau_{x y} / G_{\mathrm{VH}} \cdot \text {. . . . . . . . . . . }
\end{aligned}
$$

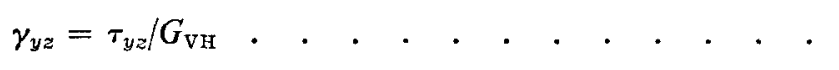

$$
\begin{aligned}
& \gamma_{z x}=2\left(1+\nu_{\mathrm{HH}}\right) \tau_{z x} / E_{\mathrm{H}} \quad \cdot \quad \cdot \quad \cdot \quad \cdot \quad \cdot \quad \cdot \quad \cdot \text {. . }
\end{aligned}
$$

We shall be concerned here only with an incompressible material and if the first three equations are added the following expression relating the volumetric strain to the stresses is obtained

$$
\frac{\Delta V}{V_{\mathrm{o}}}=e_{x}+c_{y}+e_{z}=\left(1-\nu_{\mathrm{HV}}-\nu_{\mathrm{HH}}\right) \frac{\sigma_{x}}{E_{\mathrm{II}}}+\left(\frac{E_{\mathrm{H}}}{E_{\mathrm{V}}}-2 \nu_{\mathrm{HV}}\right) \frac{\sigma_{y}}{E_{\mathrm{H}}}+\left(1-\nu_{\mathrm{HV}}-\nu_{\mathrm{HH}}\right) \frac{\sigma_{z}}{E_{\mathrm{H}}} \quad .
$$

The condition of incompressibility requires that this expression is zero for all values of the stresses; this leads to just two relations ${ }^{4}$ connecting the elastic constants, namely

$$
\begin{aligned}
& \nu_{\mathrm{HV}}=\frac{1}{2} \frac{E_{\mathrm{H}}}{E_{\mathrm{V}}} \cdot \cdot \cdot \cdot \cdot \cdot \cdot \cdot \cdot \cdot \cdot \cdot \cdot \cdot \cdot \cdot \\
& \nu_{\mathrm{HH}}=1-\frac{1}{2} \frac{E_{\mathrm{H}}}{E_{\mathrm{V}}}
\end{aligned}
$$

Incompressibility therefore reduced the five constants to three, but although $E_{\mathrm{V}}, E_{\mathrm{H}}$ and $G_{\mathrm{VH}}$ are independent of each other they cannot take arbitrary values. To examine this point further we return to the case of isotropic elasticity.

Consider the three cases of extensional, isotropic and simple shear loading of an isotropic elastic material, shown in Fig. 9. When the dotted lines refer to the unloaded form of the body, the full lines to the final configuration and the arrows point in the direction of the applied loads or stresses, this is contrary to our expectations.

If a weight $W$ is applied slowly to the end of the bar shown in the upper diagram it is unlikely ${ }^{5}$ to rise, for if it did the weight would have extracted work from the bar to increase its

4 For a material in which $E_{\mathrm{H}}=E_{\mathrm{V}}$ these reduce to a single condition $\nu_{\mathrm{HV}}=\nu_{\mathrm{HH}}=\frac{1}{2}$.

${ }^{5} \mathrm{I}$ am indebted to Professor Bishop for reminding me that materials exhibiting creep properties and capable of remembering their past stress history exist, and can behave in this way. 

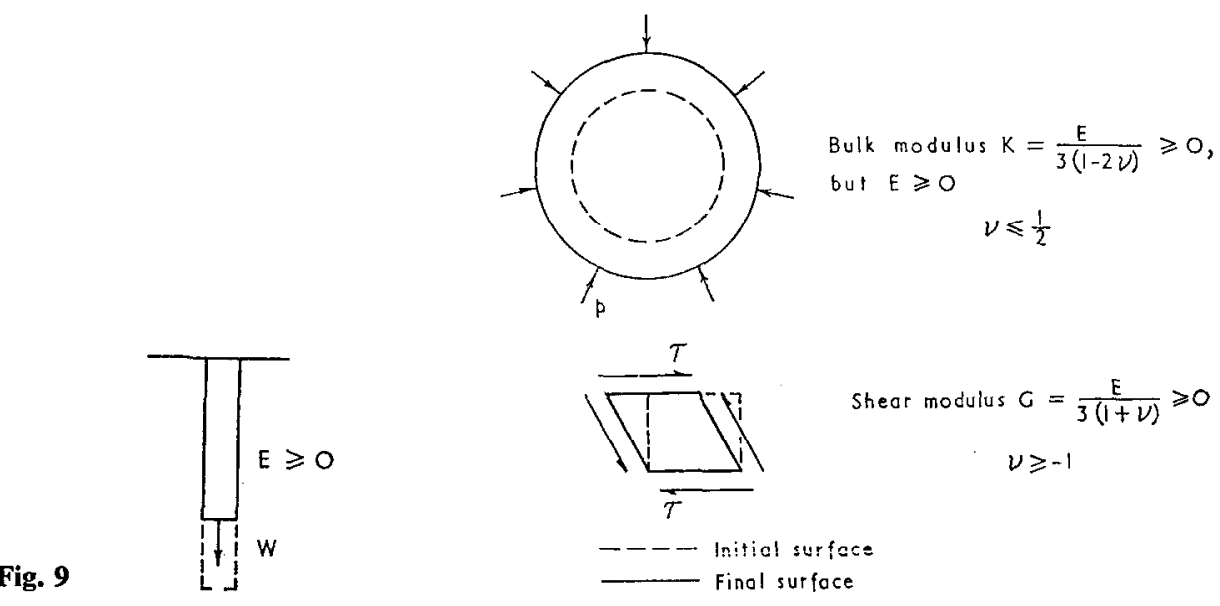

own potential energy. We conclude from this example that

$$
E \geqslant 0
$$

Similarly, a sphere subjected to an ambient pressure $p$ will not increase in volume, and this establishes that

$$
\nu \leqslant \frac{1}{2}
$$

Finally, if an element is loaded by pure shear stresses it will not choose to shear in a direction opposing the stress and therefore

$$
\nu \geqslant-1
$$

Evidently the isotropic elastic moduli must assume values which ensure that whatever combinations of stress are applied no work is extracted from the system.

The restrictions on the moduli for the special stress systems examined can be derived from the single unifying requirement that the strain energy density function $W$ is non-negative for all possible states of stress. For the isotropic case this takes the form

$$
\begin{aligned}
6 E W=(1+\nu)\left[\left(\sigma_{x}-\sigma_{y}\right)^{2}\right. & \left.+\left(\sigma_{x}-\sigma_{z}\right)^{2}+\left(\sigma_{y}-\sigma_{z}\right)^{2}\right] \\
& +(1-2 \nu)\left(\sigma_{x}+\sigma_{y}+\sigma_{z}\right)^{2}+6(1+\nu)\left(\tau_{x y}{ }^{2}+\tau_{x z}{ }^{2}+\tau_{y z}{ }^{2}\right) \quad . \quad . \quad .
\end{aligned}
$$

and it can be seen by inspection that the requirement is met in general if $-1 \leqslant \nu \leqslant \frac{1}{2}$ and $E \geqslant 0$.

For an incompressible, orthotropic, elastic medium the strain energy density $W$ is found to be given by

$$
\begin{aligned}
4 E_{\mathrm{H}} W=n\left(\sigma_{x}-\sigma_{y}\right)^{2}+(2-n)\left(\sigma_{x}-\sigma_{z}\right)^{2} & +n\left(\sigma_{y}-\sigma_{z}\right)^{2} \\
& +2 E_{\mathrm{H}} / G_{\mathrm{VH}}\left(\tau_{x y}{ }^{2}+\tau_{y z}{ }^{2}\right)+8\left(1-\frac{n}{4}\right) \tau_{z x}{ }^{2} .
\end{aligned}
$$

where

$$
n=E_{\mathrm{H}} / E_{\mathrm{V}}
$$

and $W$ can be shown to be non-negative ${ }^{6}$ in general (see, for example, Ferrar, 1941) only if

$$
\begin{aligned}
E_{\mathrm{V}}, E_{\mathrm{H}} & \geqslant 0 \\
G_{\mathrm{VH}} & \geqslant 0
\end{aligned}
$$

${ }^{6}$ Examination of the coefficients on the right-hand side suggests the condition can be met if $0 \leqslant n \leqslant 2$. This is sufficient but not necessary. Note that the three independent direct stresses appear only as two linearly independent differences. 
Fig. 10

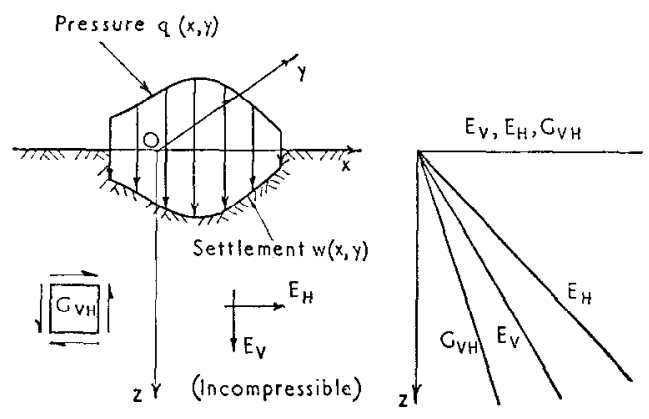

and

$$
0 \leqslant \frac{E_{\mathrm{H}}}{E_{\mathrm{V}}} \leqslant 4 \quad \cdot \quad \cdot \quad \cdot \quad \cdot \quad \cdot \quad \cdot \quad \cdot \quad \cdot \quad \cdot \quad \cdot
$$

The problem of the linearly heterogeneous, orthotropic, incompressible elastic half-space has recently been examined in some detail (Gibson and Sills, 1974; Gibson and Kalsi, 1974). It is found that if the three independent moduli $E_{\mathrm{V}}, E_{\mathrm{H}}$ and $G_{\mathrm{VH}}$ each increase linearly with depth from zero at the surface of the medium, then the simple spring behaviour is again revealed (Fig. 10). In particular, the settlement $w(x, y)$ of the plane surface ${ }^{7}$ resulting from a general surface pressure $q(x, y)$ is

$$
w(x, y)=\frac{q(x, y)}{N\left(\mathrm{~d} E_{\mathrm{V}} / \mathrm{d} z\right)} \cdot \quad \cdot \quad . \quad . \quad . \quad . \quad . \quad .
$$

As the simple orthotropy considered involves no characteristic length, this result might have been anticipated by appeal to the similarity argument. The parameter $N$ is found to be a complicated function of $E_{\mathrm{H}} / E_{\mathrm{V}}$ and $G_{\mathrm{VH}} / E_{\mathrm{V}}$, but the simple expression

$$
N=\left(G_{\mathrm{VH}} / E_{\mathrm{V}}\right)+\left(4-E_{\mathrm{H}} / E_{\mathrm{V}}\right)^{-1} \text {. }
$$

is in error by less than two per cent. It may be noted that for an incompressible isotropic material $E_{\mathrm{H}}=E_{\mathrm{V}}$ and $G_{\mathrm{VH}}=E / 3$, and equation (20) reduces to $N=\frac{2}{3}$ which agrees with the result $A=3 / 2$ recorded earlier for this case.

Owing to the restrictions which the strain energy function imposes, the first term cannot be less than zero, or the second term less than $\frac{1}{4}$ and therefore the greatest value of $N^{-1}$ is 4 . If this is compared with the factor $1 \frac{1}{2}$ obtained for the isotropic case it may be concluded that whatever the degree of anisotropy the settlement cannot exceed that experienced by an isotropic elastic clay by a factor of more than $2 \frac{2}{3}$. However, at the limit $E_{\mathrm{H}}=4 E_{\mathrm{V}}$ the material becomes rigid and no settlement occurs.

In order to evaluate $N$ in the general case, data on the independent shear modulus $G_{\mathrm{VH}}$, as well as on the two Young's moduli $E_{\mathrm{V}}$ and $E_{\mathrm{H}}$, must be available. Undrained compression tests on specimens cut with their axes in a horizontal or vertical direction will provide reliable ${ }^{8}$ data only on the Young's moduli. However, a compression test conducted on a sample cut with its axis inclined at some angle $\theta$ to the horizontal will allow Young's modulus $\left(E_{\theta}\right)$ in this direction to be measured. For an incompressible material $E_{\theta}$ is connected with the other moduli by the equation

7 The $z$ axis is directed downwards to conform with the earlier notation.

${ }^{8}$ Observations of the differential radial strains developed in a specimen cut with its axis horizontal are likely to provide unreliable information about $G_{\mathrm{VH}}$. 


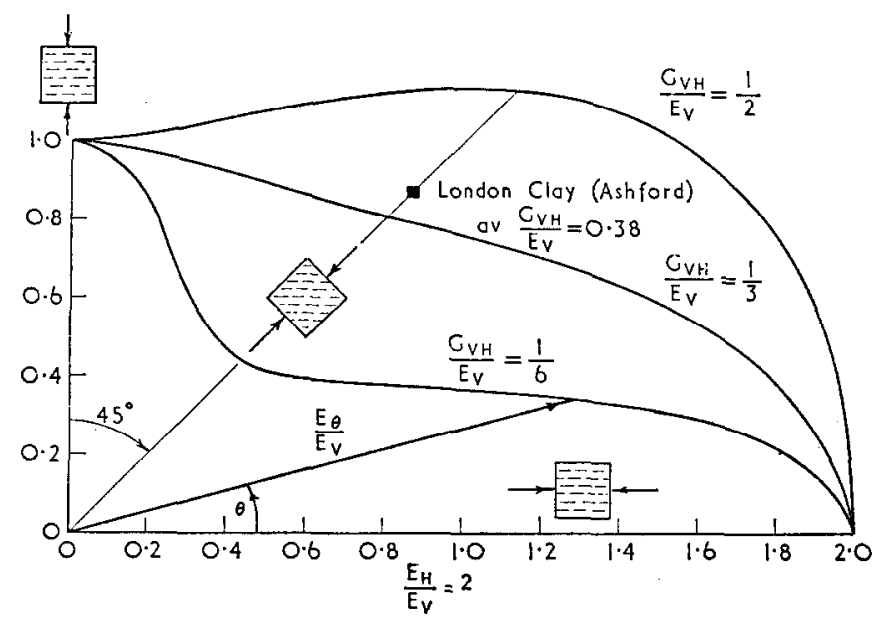

Fig. 11. Variation of undrained Young's modulus with inclination of specimen

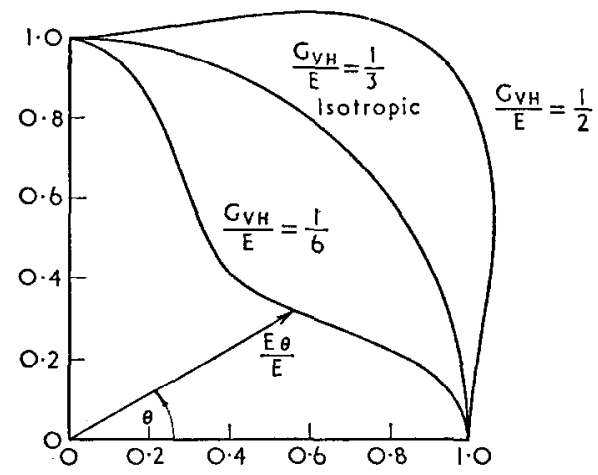

Fig. 12. Typical variations of undrained Young's modulus $\left(E_{\theta}\right)$ with orientation $(\theta)$ when $E_{\mathrm{H}}=E_{\mathrm{V}}=E$

$$
E_{0}^{-1}=\left(G_{\mathrm{VII}}^{-1}-E_{\mathrm{V}}^{-1}\right) \sin ^{2} \theta \cos ^{2} \theta+E_{\mathrm{V}}^{-1} \sin ^{4} \theta+E_{\mathrm{H}}^{-1} \cos ^{4} \theta
$$

and if $E_{\mathrm{V}}, E_{\mathrm{H}}$ and $E_{\theta}$ at some other angle-and an obvious choice is $45^{\circ}$-are measured, then the shear modulus $G_{\mathrm{VH}}$ can be found directly from this equation. The angular variation of $E_{\theta} / E_{\mathrm{v}}$ is shown in Fig. 11 when the clay is twice as stiff horizontally as vertically, and this corresponds, roughly, to what has been observed for undisturbed London Clay. Three values of the ratio of shear modulus to vertical Young's modulus, namely

$$
\frac{G_{\mathrm{VH}}}{E_{\mathrm{V}}}=\frac{1}{6}, \frac{1}{3}, \frac{1}{2}
$$

have been taken, the middle value being appropriate for an incompressible isotropic material. The influence of this parameter on the modulus at $45^{\circ}$, for example, is very pronounced, which confirms that this inclination is a natural one to choose for determining the shear modulus.

It will be clear from equation (21) that it is possible for a material to exist which although possessing the same vertical and horizontal moduli is, nevertheless, not isotropic. The angular variation of $E_{\theta} / E$ in this rather special material, which might be termed 'pseudoisotropic', is illustrated in Fig. 12. There is an eightfold symmetry about the planes inclined at $45^{\circ}$, the greatest or least modulus occurring in these directions. Therefore, although 
equality of the vertical and horizontal Young's moduli is necessary to ensure isotropy it is not alone sufficient-in addition, the shear modulus in an incompressible material must assume a value equal to one third of $E$.

In this connexion it is of interest to examine data by Dr Ward and his colleagues of the Building Research Station obtained from laboratory undrained compression tests on undisturbed samples of London Clay cut from blocks secured from shafts and tunnels. The stress history to which London Clay has been subjected would be expected to have developed marked anisotropy and we find this to be the case. Table 1 records values of the ratio $E_{\mathrm{H}} / E_{\mathrm{V}}$ measured on samples obtained from eight sites in the London area designated by the code letters used by

Table 1. Ratio $E_{\mathrm{H}} / E_{\mathrm{v}}$ of undrained Young's moduli of undisturbed London Clay (after Ward, Samuels and Butler, 1959)

\begin{tabular}{l|c|c|c}
\hline \multirow{2}{*}{ Site } & \multicolumn{3}{|c}{$E_{\mathrm{H}} / E_{\mathrm{v}}$} \\
\cline { 2 - 4 } & First loading & Unloading & Reloading \\
\hline $\mathrm{L}$ & 1.7 & 1.7 & 1.6 \\
$\mathrm{~K} 1$ & 1.9 & 1.8 & 1.8 \\
$\mathrm{~K} 2$ & 2.0 & 2.0 & 1.9 \\
$\mathrm{G}$ & 1.1 & 1.4 & 1.8 \\
$\mathrm{~B} 1, \mathrm{~B} 3$ & 1.9 & 2.0 & 1.9 \\
$\mathrm{~B} 2$ & 1.6 & 1.4 & 1.4 \\
$\mathrm{O}$ & 1.2 & 1.3 & 1.3 \\
T & 1.4 & 1.5 & 1.5 \\
Average & 1.60 & 1.64 & 1.65 \\
\hline
\end{tabular}

Table 2. Undrained compression test data on London Clay (after Ward, Marsland and Samuels, 1965)

\begin{tabular}{|c|c|c|c|c|c|}
\hline$\underset{\mathrm{ft}}{\text { Depth, }}$ & Orientation* & $\begin{array}{l}\text { Average undrained secant } \\
\text { modulus at half ultimate, } \\
\qquad / / \mathrm{ft}^{2}\end{array}$ & $\begin{array}{l}\text { No. of } \\
\text { samples }\end{array}$ & $E_{\mathrm{H}} / E_{\mathrm{V}}$ & $G_{\mathrm{VH}} / E_{\mathrm{V}}$ \\
\hline 30 & $\begin{array}{l}\mathrm{V} \\
\mathrm{H} \\
\mathrm{D}\end{array}$ & $\begin{array}{l}372 \\
504 \\
410\end{array}$ & $\begin{array}{l}28 \\
24 \\
14\end{array}$ & $1 \cdot 35$ & $0 \cdot 35$ \\
\hline 50 & $\begin{array}{l}\mathrm{V} \\
\mathrm{H} \\
\mathrm{D}\end{array}$ & $\begin{array}{l}366 \\
581 \\
440\end{array}$ & $\begin{array}{r}25 \\
8 \\
4\end{array}$ & $1 \cdot 59$ & $0 \cdot 37$ \\
\hline 66 & $\begin{array}{l}\mathrm{V} \\
\mathrm{H} \\
\mathrm{D}\end{array}$ & $\begin{array}{l}394 \\
820 \\
552\end{array}$ & $\begin{array}{l}30 \\
20 \\
13\end{array}$ & $2 \cdot 08$ & 0.42 \\
\hline 91 & $\begin{array}{l}\mathrm{V} \\
\mathrm{H} \\
\mathrm{D}\end{array}$ & $\begin{array}{r}448 \\
1060 \\
670\end{array}$ & $\begin{array}{r}18 \\
8 \\
3\end{array}$ & $2 \cdot 37$ & 0.44 \\
\hline 114 & $\begin{array}{l}\mathrm{V} \\
\mathrm{H} \\
\mathrm{D}\end{array}$ & $\begin{array}{r}786 \\
1358 \\
1103\end{array}$ & $\begin{array}{l}47 \\
21 \\
15\end{array}$ & $1 \cdot 73$ & 0.44 \\
\hline 138 & $\begin{array}{l}\mathrm{V} \\
\mathrm{H} \\
\mathrm{D}\end{array}$ & $\begin{array}{l}1383 \\
2687 \\
1137\end{array}$ & $\begin{array}{r}17 \\
12 \\
5\end{array}$ & 1.94 & $0 \cdot 23$ \\
\hline \multicolumn{4}{|r|}{ Average } & $1 \cdot 84$ & $0 \cdot 38$ \\
\hline
\end{tabular}

${ }^{*} \mathrm{~V}-$ Vertical, H-Horizontal, D-Inclined at $45^{\circ}$. 


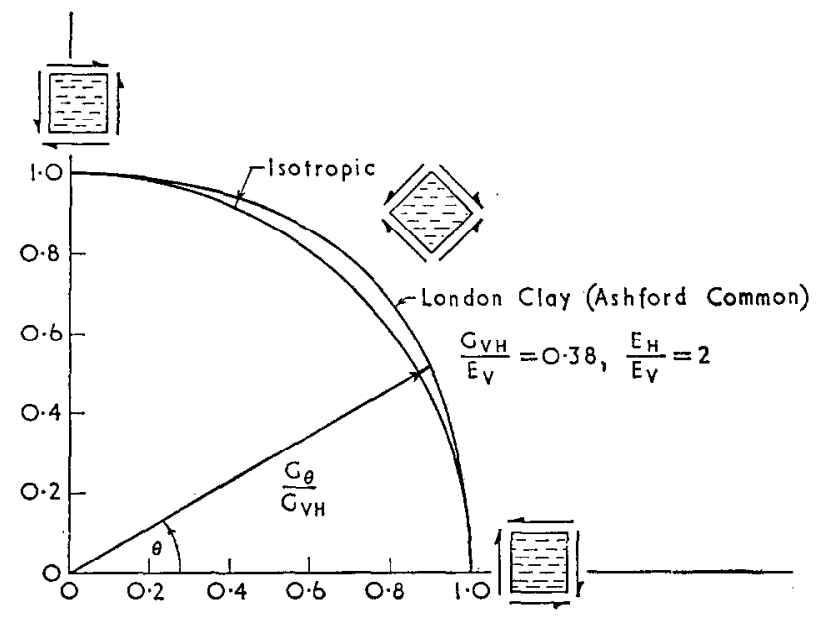

Fig. 13. Variation of shear modulus with orientation of specimen

Ward, Samuels and Butler in their paper (Ward et al., 1959). The sites extend from Hampstead to Stockwell and from Walton to the City. It is significant that although the samples were obtained from a range of depths and a variety of locations, and the moduli themselves were about $30 \%$ lower on first loading than in the subsequent cycles of unloading and reloading, yet the ratio of the moduli presents a reasonably consistent pattern with an average value a little greater than 1.6. Skempton and Henkel (1957) estimate that between 500 and $700 \mathrm{ft}$ of overburden has melted or been eroded from the area of central London, but despite this appreciable preconsolidation pressure less than one half of the theoretical upper limit of induced anisotropy of 4 has been attained. Special features at the various sites can explain some of the variations in Table 1-for example, the high degree of fissuring observed in samples from site G-and these points have been discussed in detail by the authors, but the overall picture is quite clear.

Data from more than three hundred undrained compression tests on specimens cut from block samples of London Clay obtained from various depths at Ashford Common were published subsequently (Ward et al., 1965) and this has been used in Table 2. Undrained tests were conducted on samples cut vertically, horizontally and inclined at $45^{\circ}$ and these are referred to by the symbols V, H and D. The ratio of horizontal to vertical modulus is some $20 \%$ higher on average here and at depths of 66 and $91 \mathrm{ft}$ the ratio exceeds 2 . This greater degree of anisotropy may reflect the greater pressures at Ashford which Bishop et al.(1965) estimated to correspond to $1200-1300 \mathrm{ft}$ of overburden. There is a slight tendency for the ratio to increase with depth, but whether this points to some recovery of isotropic behaviour in the upper part of the stratum where the overconsolidation ratio is greater than it is at depth, or whether it is due to the presence of more open joints and fissures at these shallow depths, remains an open question.

The compression tests on inclined specimens allow the shear modulus $G_{\mathrm{VH}}$ to be found from equation (21). Although there is some depth variation and scatter, the average value as a ratio of the vertical modulus is 0.38 , which exceeds that which would hold in the isotropic case by only $15 \%$. It must be emphasized that $G_{\mathrm{VH}}$ is the shear modulus which would be found if shear stresses were applied to the vertical and horizontal faces of an element in situ. If the same system of stress were applied to an element with faces oriented at an angle $\theta$ to the horizontal (or vertical) direction, then a shear modulus $G_{\theta}$ would be measured and this differs, 
in general, from $G_{\mathrm{VH}}$. As the axes of reference rotate with respect to the material, the components of stress and strain transform according to the geometry of Mohr's circle, but the components of the elasticity tensor, which is symmetric rank four, transform according to more complicated rules. These considerations, which have led to equation (21), show that $G_{\theta}$ is given by

$$
\frac{G_{\theta}}{G_{\mathrm{VH}}}=\left[\cos ^{2} 2 \theta+\frac{G_{\mathrm{VH}}}{E_{\mathrm{V}}}\left(\frac{E_{\mathrm{V}}}{E_{\mathrm{H}}}+2\right) \sin ^{2} 2 \theta\right]^{-1} .
$$

This relation allows the variation of $G_{\theta}$ with specimen orientation to be calculated. Taking $G_{\mathrm{VH}} / E_{\mathrm{V}}=0.38$ and $E_{\mathrm{H}} / E_{\mathrm{V}}=2$ as typical for undisturbed London Clay, it is found that rotation of the reference axes is accompanied by a very small variation in the shear modulus (Fig. 13) amounting to no more ${ }^{9}$ than $5 \%$, although the Young's moduli differ by a factor 2 .

Having considered the extensions of classical elasticity theory which are needed to take account of anisotropy and heterogeneity, and these factors in relation to the undrained deformation properties of London Clay, it is appropriate to give some attention to their implications. Accordingly, we examine the influence of three factors, namely

(a) layer depth,

(b) elastic heterogeneity,

(c) elastic orthotropy

on the immediate surface settlement of a layer of London Clay $110 \mathrm{ft}$ thick resting on a rough rigid base. The loading is a vertical pressure of 1 ton $/ \mathrm{ft}^{2}$ distributed uniformly over a circular area of radius $110 \mathrm{ft}$. For this calculation the data on $E_{\mathrm{V}}$ and $E_{\mathrm{H}}$ from Ashford Common, which is shown in Fig. 14, have been used. The full lines (partial heterogeneity) show the best

Table 3. Influence of stratum depth, elastic heterogeneity and orthotropy on settlement of circular $(R=110 \mathrm{ft})$ loaded area $\left(q=1 \mathrm{t} / \mathrm{ft}^{2}\right)$ on surface of London Clay

\begin{tabular}{|c|c|c|c|c|c|c|c|c|}
\hline \multirow{2}{*}{$\begin{array}{l}\text { Stratum } \\
\text { depth, } \\
\text { ft }\end{array}$} & \multirow{2}{*}{$E_{\mathrm{H} /} / E_{\mathrm{V}}$} & \multirow{2}{*}{$\begin{array}{c}E_{\mathrm{V}}(\mathrm{o}) \\
\mathrm{t} / \mathrm{ft}^{2}\end{array}$} & \multirow{2}{*}{$\stackrel{\lambda}{\mathbf{t} / \mathrm{ft}^{2}}$} & \multirow{2}{*}{$G_{\mathrm{VH}} / E_{\mathrm{V}}$} & \multicolumn{3}{|c|}{ Settlement, $\mathrm{ft}$} & \multirow{2}{*}{$\begin{array}{l}\text { Factors taken into } \\
\text { account }\end{array}$} \\
\hline & & & & & Centre & Edge & Centre-Edge & \\
\hline$\infty$ & $1 \cdot 0$ & 500 & 0 & $0 \cdot 33$ & $0 \cdot 322$ & $0 \cdot 205$ & $0 \cdot 177$ & - \\
\hline$\infty$ & $1 \cdot 8$ & 500 & 0 & $0 \cdot 38$ & $0 \cdot 238$ & $0 \cdot 153$ & $0 \cdot 085$ & Orthotropy \\
\hline$\infty$ & $1 \cdot 0$ & 0 & $9 \cdot 1$ & $0 \cdot 33$ & $0 \cdot 165$ & $0 \cdot 165$ & 0 & Full heterogeneity \\
\hline$\infty$ & $1 \cdot 0$ & 200 & $5 \cdot 5$ & $0 \cdot 33$ & $0 \cdot 171$ & 0.087 & $0 \cdot 084$ & Partial heterogeneity \\
\hline$\infty$ & $1 \cdot 8$ & 0 & $9 \cdot 1$ & $0 \cdot 38$ & $0 \cdot 132$ & $0 \cdot 132$ & 0 & $\begin{array}{l}\text { Orthotropy, } \\
\text { full heterogeneity }\end{array}$ \\
\hline 110 & $1 \cdot 0$ & 500 & 0 & $0 \cdot 33$ & $0 \cdot 101$ & 0.031 & 0.070 & Depth \\
\hline 110 & $1 \cdot 8$ & 500 & 0 & $0 \cdot 38$ & 0.072 & 0.022 & 0.050 & Depth, orthotropy \\
\hline 110 & $1 \cdot 0$ & 0 & $9 \cdot 1$ & $0 \cdot 33$ & $0 \cdot 112$ & $0 \cdot 131$ & -0.019 & $\begin{array}{l}\text { Depth, } \\
\text { full heterogeneity }\end{array}$ \\
\hline 110 & $1 \cdot 8$ & 0 & $9 \cdot 1$ & $0 \cdot 38$ & 0.080 & 0.093 & -0.013 & $\begin{array}{l}\text { Depth, orthotropy, } \\
\text { full heterogeneity }\end{array}$ \\
\hline
\end{tabular}

${ }^{8}$ It would be interesting if this insensitivity proved to be a general feature of clays (see Barden, 1963). 


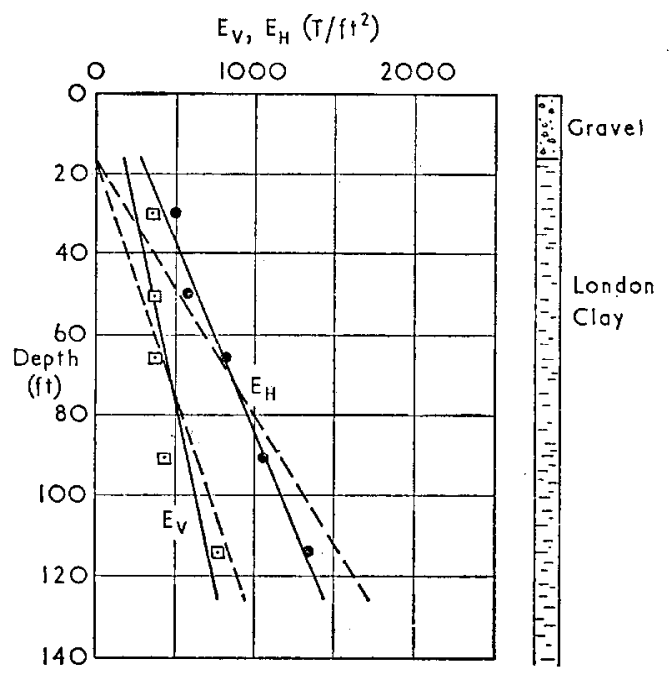

Fig. 14. Depth variation of vertical and horizontal undrained Young's moduli of London Clay (Ashford Common) (after Ward, Marsland and Samuels, 1965)

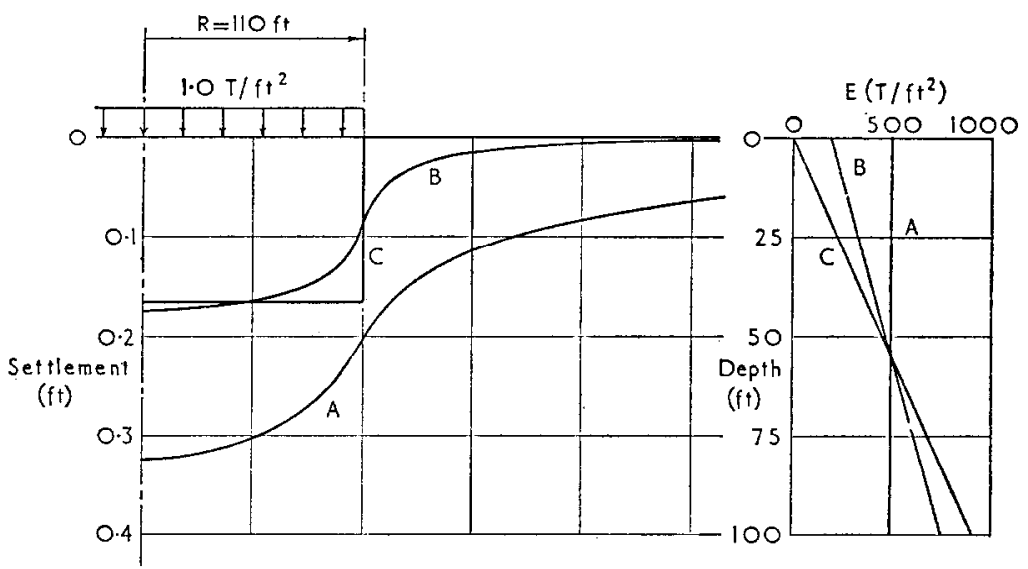

Fig. 15. Influence of elastic heterogeneity on surface immediate settlement profile (isotropic incompressible stratum of infinite depth)

linear fit through the individual points, while the dashed lines (full heterogeneity) give vanishing surface $^{10}$ moduli and here the agreement with the observations is less good.

The properties of the clay are shown in Table 3, together with the calculated immediate settlements at the centre and edge of the loaded area. For those cases where the depth heterogeneity is ignored, the average values of the moduli through the full layer depth of $110 \mathrm{ft}$ have been assumed in the calculation. The first entry in Table 3 relates to the simplest assessment where the clay layer is replaced by a uniform, isotropic elastic half-space: the vertical modulus $E_{\mathrm{V}}$ is used in the calculation as this is usually employed. All the factors have been included in the last entry and when this is compared with the simple calculation it is seen that the centre settlement has decreased by $75 \%$, the edge settlement by $50 \%$, while the differential settlement between centre and edge has declined by an order of magnitude and changed its sign.

The effect of introducing heterogeneity alone is shown in Fig. 15. As the variation of $E\left(=E_{\mathrm{v}}\right)$ with depth changes from a uniform distribution (line $\left.\mathrm{A}\right)$ to line $\mathrm{B}$ (partial heterogeneity)

${ }^{10}$ The loading is applied directly to the surface of the clay at a depth of $18 \mathrm{ft}$. 
the reduction in average settlement resulting from the increased stiffness of the clay below about $50 \mathrm{ft}$ is not offset by the reduction of $E$ above this level. The change from partial to complete heterogeneity (line B to line $\mathrm{C}$ ) hardly affects the average settlement, but the differential settlement is concentrated at the edge as a step.

When the limited depth of the layer is also allowed for (Fig. 16) the heterogeneity increases slightly the average settlement, but the settlement profile is again radically altered.

Stratum depth is, of course, commonly taken into account in settlement calculations, but the influence of orthotropy, which is tantamount to using a value of $E$ greater than $E_{\mathrm{V}}$, is not negligible, while the heterogeneity has a pronounced effect on the edge settlement.

Apart from its simplicity this example departs from reality in another important respect. The ground is rarely loaded directly but usually through a foundation possessing some flexural rigidity. The surface of the soil then experiences not the pressure applied at the top of a raft but the counter-pressure on its base which will, in general, be distributed quite differently. The counter-pressure adjusts itself in such a way that the bent form of the raft just matches the deformed surface of the soil. The earliest attempts to develop a reasonable procedure for determining bending moments and shearing forces in a raft stem from the work of Winkler (1867) who assumed, in effect, that the soil responded to surface loading like a bed of springs. This assumption was first used in the design of rail track and later found wide application as the theory of subgrade reaction. Although much has been written wholly devoted to the consequences of assuming the validity of this simple model (Hayashi, 1921; Hetényi, 1946), it has come under strong criticism. The virtue of the model lay in its simplicity, but later, when it was clearly recognized that uniform pressures do not produce uniform settlements, various attempts were made to improve it either by adjusting the coefficient of subgrade reaction over the base of the foundation so that observed settlement patterns could be matched, or introducing diagonal springs in the model subgrade to make it capable of spreading load like a continuum. Although understandable, these efforts were, in my view, misdirected. Appropriate parameters had to be selected for each circumstance and the model conspicuously lacked what all models should possess-predictive power.

Reluctance to abandon this model stemmed partly from the mathematical difficulties which were encountered when soil is represented by an elastic continuum. Although in 1885 Boussinesq had solved the problem of a symmetrically loaded rigid circular raft resting on an elastic half-space, more than fifty years passed before Borowicka (1936) succeeded in generalizing the result to a raft of finite stiffness.

Between these two models lies the heterogeneous elastic continuum. At one extreme it behaves like the Winkler model and at the other like Boussinesq's and Borowicka's uniform elastic continuum. Dr Brown of Sydney University has recently succeeded in extending Borowicka's work to take account of the increasing stiffness of the ground with depth (Brown, 1969, 1972). Fig. 17 shows how this affects the distribution of reaction pressure on the base of a uniformly loaded rigid raft. The ratio of the surface modulus $E(0)$ to that at a depth equal to the radius $R$ of the raft is a convenient measure of heterogeneity; when it is unity Boussinesq's solution is recovered and when zero Winkler's distribution is obtained. The reaction pressure distributions are rcminiscent of those given by Borowicka for different raft flexibilities and it is evident that raft flexibility and soil heterogeneity augment each other, so that Borowicka's solution tends to overestimate raft bending moments while Winkler's method underestimates them-at least in examples of simple loading such as this. The important influence of soil heterogeneity on the maximum bending moment, ${ }^{11}$ which occurs

11 Whether or not the symmetric bending assumed here will hold for all values of the relative rigidity of soil and or raft, whether asymmetric bending can develop under uniform pressure in some cases is an open question. 


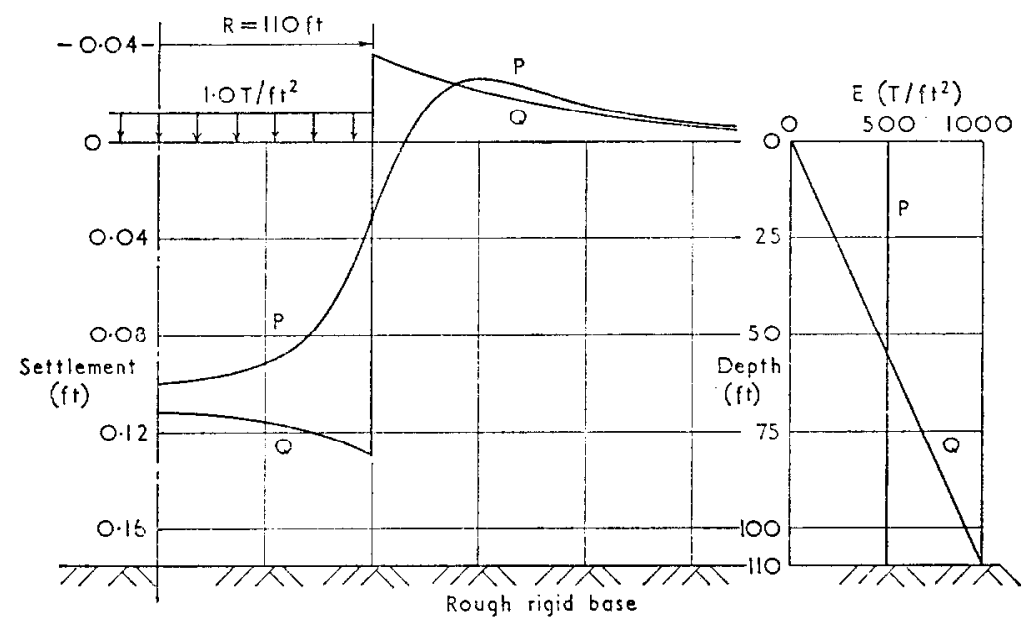

Fig. 16. Influence of elastic heterogeneity on surface immediate settlement profile (isotropic incompressible layer, depth $110 \mathrm{ft}$ )

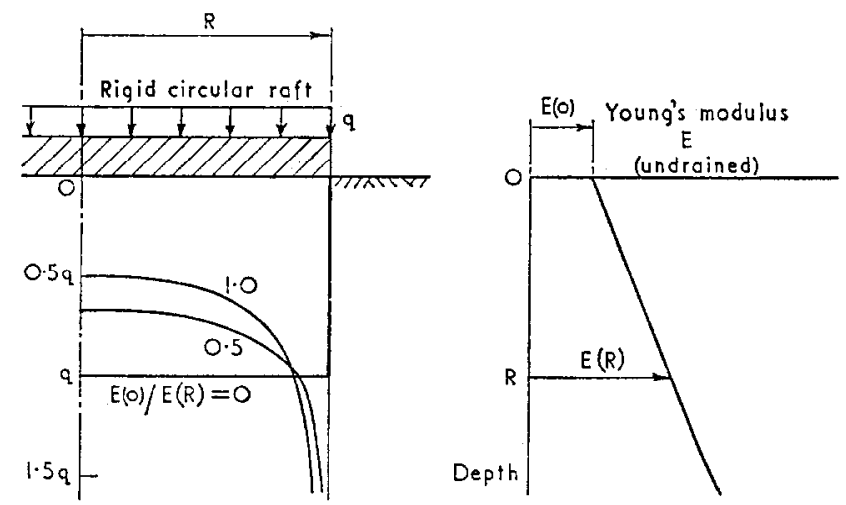

Fig. 17. Influence of soil heterogeneity on reaction pressure distribution beneath uniformly loaded rigid circular raft (incompressible, isotropic elastic half-space) (after Brown, 1972)

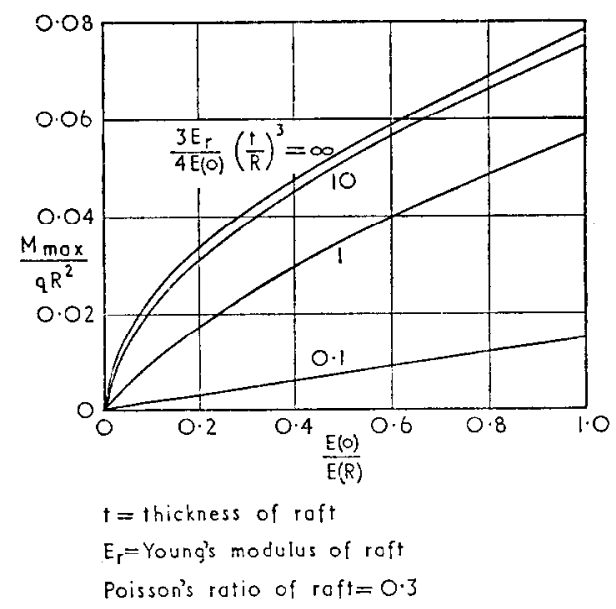

Fig. 18. Variation of maximum bending moment with degree of soil heterogeneity (incompressible, isotropic elastic half-space) (after Brown, 1972) 
Fig. 19

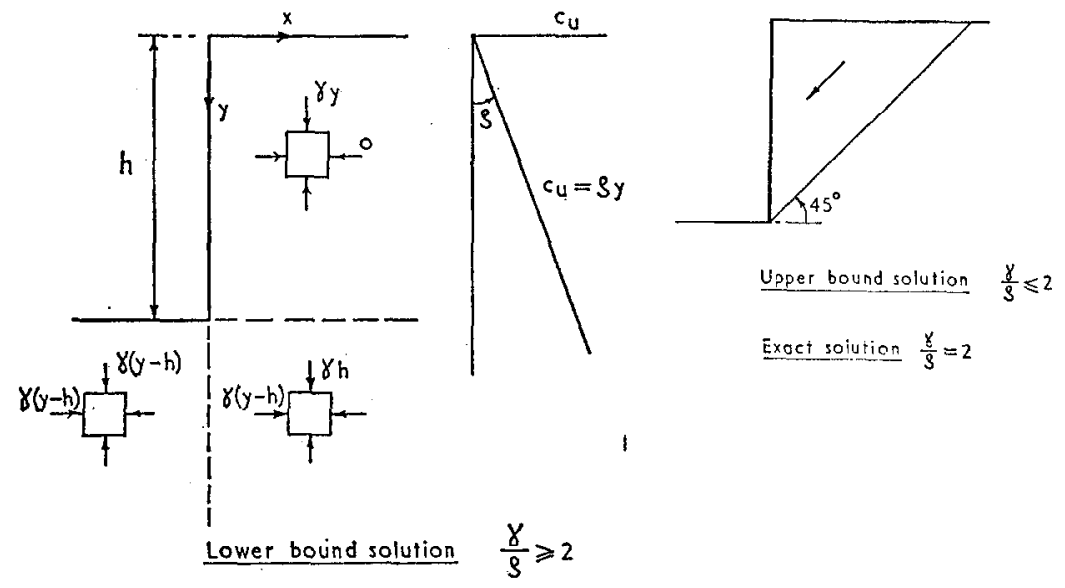

usually but not invariably at the centre of the raft, is illustrated for the same problem in Fig. 18.

\section{SLOPE STABILITY AND BEARING CAPACITY $(\phi=0)$}

The influence of heterogeneity

It is well known that the increase of undrained Young's modulus $E_{\mathrm{u}}$ with depth, which is a characteristic feature of most clays, is accompanied by a corresponding increase in the undrained shear strength $c_{\mathrm{u}}$. It is natural to enquire whether the marked influence which the former has in problems of deformation will be linked with comparable effects in problems of bearing capacity and short-term stability of slopes.

The simplest method available to examine the possible consequences of an increase of strength with depth is the $\phi=0$ slip circle analysis. It is widely recognized that this procedure provides an upper bound to the collapse load or the critical height of a cutting. Very often the 'worst' circle gives an answer of acceptable accuracy for engineering purposes and well within the range of uncertainty encountered in estimating the actual loading or the average shear strength. For example, the bearing capacity of a strip load on uniform clay is overestimated by only $8 \%$.

In some cases, notably the stability of a vertical unsupported cut, there is less certainty that the most critical circle leads to a satisfactory answer. This uncertainty arises chiefly from the lack of exact solutions to more than a few problems. However, methods have been developed in the theory of plasticity for obtaining estimates which are always on the pessimistic side. These so-called lower bound solutions (Drucker and Prager, 1952) are determined by finding a complete stress field which everywhere satisfies the requirements of equilibrium, which meets the stress boundary conditions and nowhere violates the failure criterion.

If upper bound and lower bound solutions have been obtained to a problem then at least there is certainty that the exact solution lies between the greatest lower bound and the least upper bound. When these are close our uncertainties are removed, but when despite considerable effort on our part the gap remains wide the uncertainties remain, but at least we have no false confidence in the upper bound. This is the case for a vertical cut in uniform cohesive soil (Heyman, 1973b). If the gap can be closed completely then an exact solution to the problem has been found, although neither the failure mechanism assumed in the upper bound solution nor the stress distribution in the lower bound solution may correspond to what actually happens in the ground. 

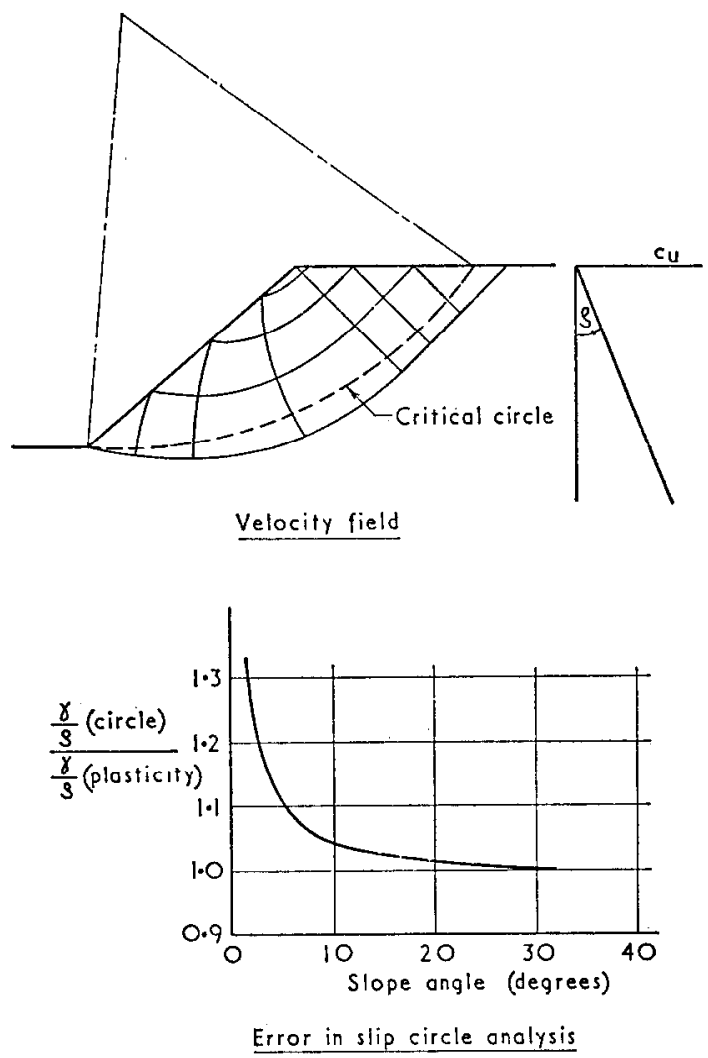

Fig. 20. Slope stability (undrained) in ideal normally consolidated clay (after Booker and Davis, 1972)

As a simple and rather artificial example let us consider whether a stable unsupported vertical face can be dug in an ideal normally consolidated clay. The undrained shear strength is assumed to increase linearly with depth from a zero at the ground surface. The lower diagram in Fig. 19 shows a possible plane failure surface inclined at $45^{\circ}$ and passing through the toe of the cut. Using simple statics, it can be easily shown that the cut will certainly not be stable unless the bulk unit weight $\gamma$ of the clay is less or equal to twice the rate of increase $\rho$ of the shear strength with depth. The upper diagram shows a stress distribution which satisfies equilibrium and the boundary conditions, although it looks far from realistic (Heyman, 1973a). The dotted lines indicate planes of stress discontinuity ${ }^{12}$ and these may be allowed as long as equilibrium is satisfied for those elements through which they pass. If $\gamma=2 \rho$ it will be seen that the clay above the toe of the cut has just reached failure and it can be checked that failure is not attained elsewhere. Since this is a lower bound solution $\gamma$ may be greater than $2 \rho$ before collapse occurs. The bounds are coincident and therefore if the cut is just stable $\gamma=2 \rho$, and if $\gamma<2 \rho$ there is some margin of safety. The depth $h$ of the cut does not enter into the solution and this implies that if a stable cut can be dug a foot deep it will remain stable at the same factor of safety ${ }^{13}$ as the depth is increased. Are any real clays likely to be stable under

\footnotetext{
12 Discontinuity or stress gradient.

${ }^{13}$ This is true as long as the undrained condition continues to hold.
} 

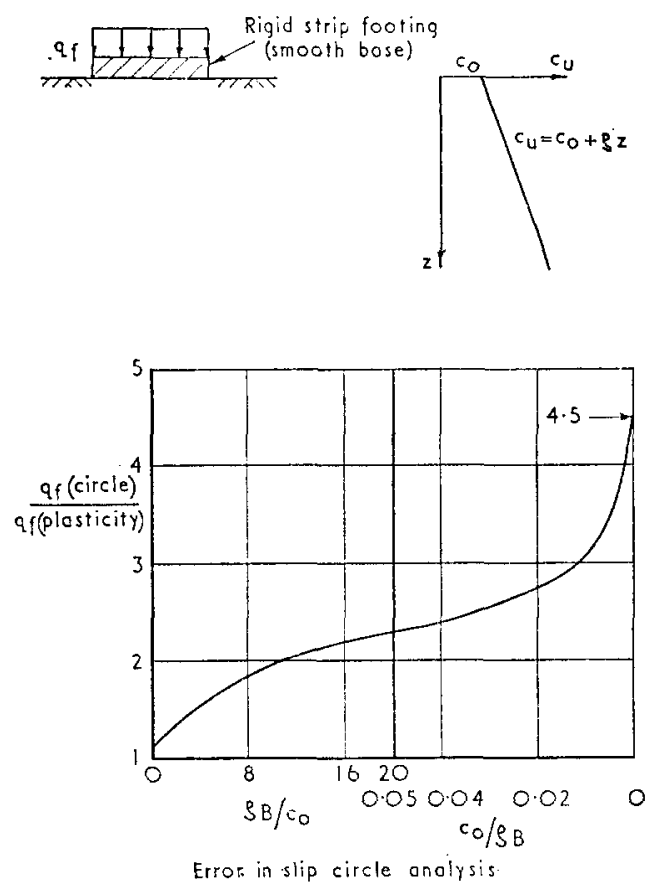

Fig. 21. Bearing capacity in normally consolidated clay (after Davis and Booker, 1973)

these conditions in the short-term? If the groundwater table is at the surface of the clay we may write

$$
\rho=\left(\gamma-\gamma_{\mathrm{w}}\right) \mathrm{d} c_{\mathrm{u}} / \mathrm{d} p^{\prime}
$$

where $p^{\prime}$ is the vertical effective consolidation pressure and $\gamma_{\mathrm{w}}$ is the unit weight of water. The cut will therefore be stable only if

$$
\mathrm{d} c_{\mathrm{u}} / \mathrm{d} p^{\prime} \geqslant \gamma / 2\left(\gamma-\gamma_{\mathrm{w}}\right) \quad \text {. . . . . . . . . }
$$

and if, as a rough approximation, ${ }^{14}$ we take $\gamma=2 \gamma_{w}$, equation (24) reduces to

$$
\mathrm{d} c_{\mathrm{u}} / \mathrm{d} p^{\prime} \geqslant 1
$$

Clays normally consolidated in nature under conditions of zero lateral strain rarely exhibit values of $\mathrm{d} c_{\mathrm{n}} / \mathrm{d} p^{\prime}$ above 0.4 and values in excess of 0.5 would be wholly exceptional (Skempton, 1970). It must, therefore, be concluded that for vertical cuts in those normally consolidated clays which do not possess a zone of desiccation close to the surface, support will always be needed.

An 'exact' numerical solution to the more general stability problem of a uniform slope cut in a clay of this type was published recently (Booker and Davis, 1972). They showed that as long as a just stable slope is not flatter than about $5^{\circ}$, the solution to this problem based on a worst circle (Gibson and Morgenstern, 1962) is very little in error. ${ }^{15}$

For this case the shape of the most critical circle corresponds in shape well with the trajectories of the velocity field they derive (Fig. 20). Once again, the stability depends only on the slope inclination and not on its height.

This agreement is comforting, but if we turn to the undrained bearing capacity of a smoothbased strip load on a clay of this type (Fig. 21) the same authors find that the worst circle gives

\footnotetext{
${ }_{14}$ If $\gamma<2 \gamma_{\pi}$ the condition on $\mathrm{d} c_{\mathrm{u}} / \mathrm{d} p^{\prime}$ becomes more severe.

15 Their solution for the vertical cut also agrees with that derived here.
} 

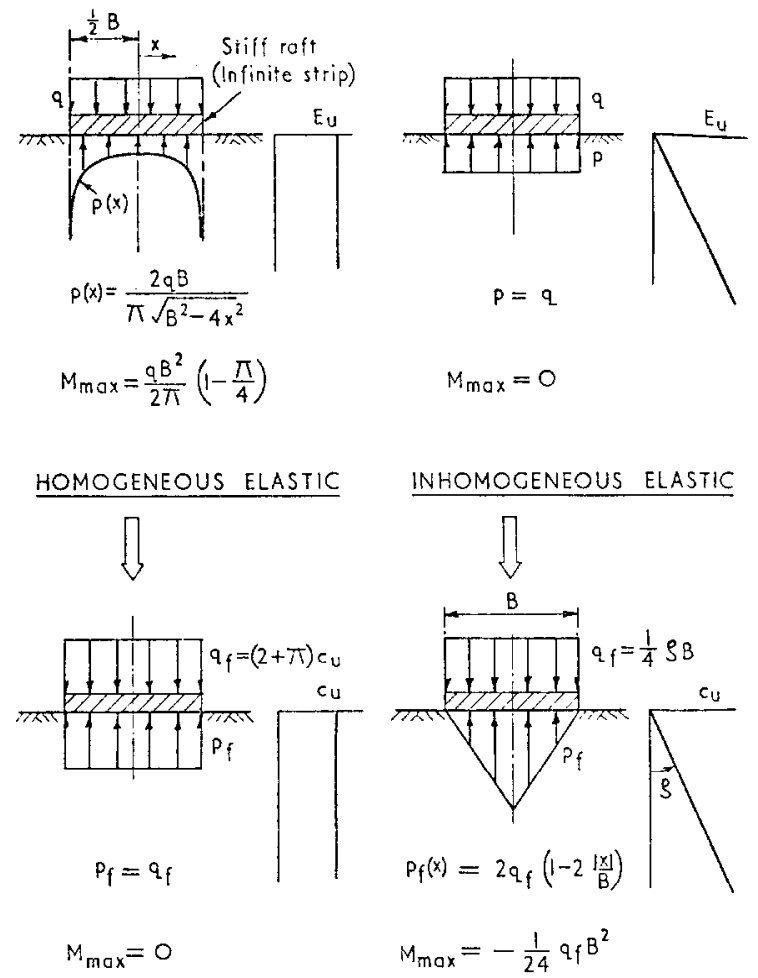

INHOMOGENEOUS ELASTIC

HOMOCENEOUS PLASTIC

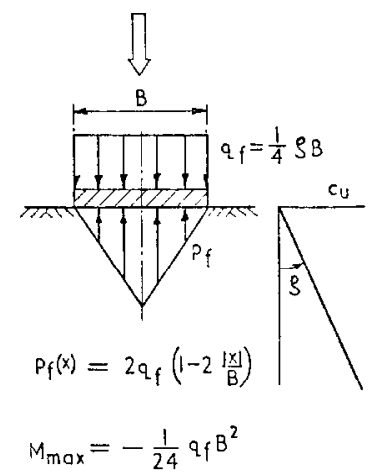

INHOMOGENEOUS PLASTIC

Fig. 22. Influence of depth heterogeneity of $E_{\mathrm{u}}$ and $c_{\mathrm{u}}$ on bending of a strip raft (smooth contact)

wildly overoptimistic results (Davis and Booker, 1973). ${ }^{16}$ Even for clays which have a reasonable surface strength the error involved in the slip circle analysis can be astonishingly high. The reason for this seems to be that no circle can be chosen to conform well with the trajectories of the velocity field. This result must be regarded as of the utmost importance and it suggests that there is a real need for further exact solutions to problems where factors such as strength heterogeneity and the inability of some clays to carry tensile stresses are examined and the results compared with simple engineering methods such as the slip circle analysis. Only in this way can we discover the factors to which the answers are really sensitive and the circumstances where these are likely to be influential.

To draw together the themes of elasticity and plasticity and the influence of simple heterogeneity to which I have drawn attention, it is instructive to examine how the maximum transverse bending moment in a uniformly loaded long strip footing alters as the loading increases and underlying clay passes from an elastic condition to a state of fully developed plasticity (Fig. 22). The two extreme cases of uniformity and of linear heterogeneity in both the undrained Young's modulus $E_{\mathrm{u}}$ and the undrained strength $c_{\mathrm{u}}$ have again been adopted. When the depth variation of this quantity can be ignored and this might be the case in a plate-loading test or for a footing of limited size at the surface of a clay stratum, the maximum bending moment increases from zero then passes through a maximum and approaches zero again as the ultimate bearing capacity is reached. When the foundation is large the increasing stiffness and strength

16 See also Livneh and Greenstein, 1973. 


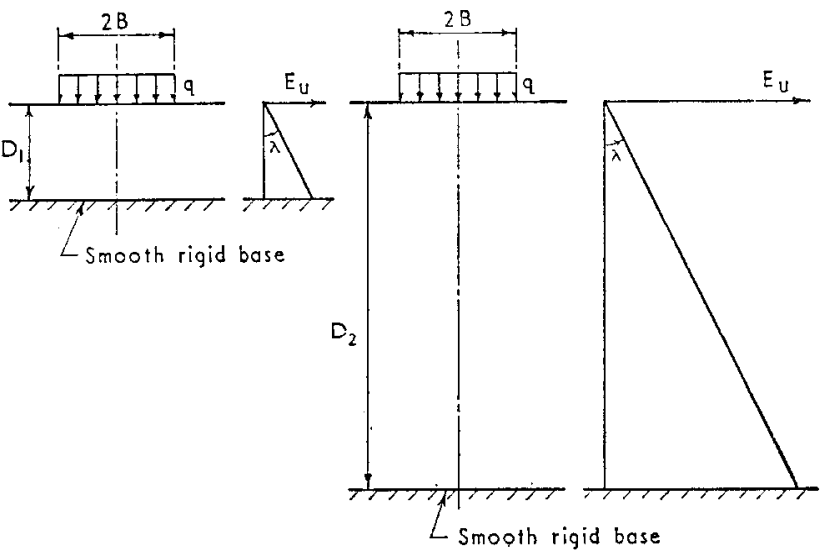

Fig. 23. Which load settles more?

of the clay with depth modifies the counter-pressure distribution in such a way that the bending moment continues to rise as the loading increases and it reaches its greatest value when the soil is about to fail. Furthermore, the sense of bending is now 'hogging' instead of 'sagging'.

\section{CONCLUSIONS}

The analytical method draws attention to broad trends and helps to distinguish between which factors are of primary significance and which are of secondary importance.

It is neither just a dispensible supplement to engineering intuition, nor merely a procedure for quantifying results.

It is able to 'speak for itself' and on occasion does so in characteristic ways which may be illustrated by the following example.

The incompressible heterogeneous elastic layer we have been concerned with earlier is subject to a uniform strip loading (Fig. 23). The layer rests on a smooth rigid base at some small depth $D_{1}$ in the diagram on the left and at some much greater depth $D_{2}$ in the diagram on the right. In all other respects these two cases are identical. If we were required to guess which load settles more, the majority would probably conclude that the load on the deeper layer does. It seems difficult to believe that this answer is incorrect, but it is. In fact an analysis by Professor Awojobi of Lagos University has shown recently that the settlement is completely independent of layer depth (Awojobi, 1974).

The settlement $S_{0}$ of any point on the surface of the incompressible layer at a distance $x$ from the centre-line of the strip load, can be expressed in the form

$$
S_{\mathrm{o}}(x)=\frac{3}{4} \int_{0}^{D} \frac{\left(\sigma_{z}-\sigma_{x}\right)}{E_{\mathrm{u}}(z)} \mathrm{d} z \quad . \quad . \quad . \quad . \quad . \quad . \quad .
$$

where the symbols have their usual meaning.

In this case

$$
E_{\mathrm{u}}(z)=\lambda z \quad \cdot \quad \text {. }
$$

and, based on Awojobi's analysis, expressions have been derived for $\sigma_{z}$ and $\sigma_{x}$ the vertical and horizontal components of stress. The stress difference is found to be given by

$$
\frac{\left(\sigma_{z}-\sigma_{x}\right)}{q}=-\frac{z}{D}\left[\frac{\sinh \pi(B+x) / D}{\cosh \pi(B+x) / D-\cos \pi z / D}+\frac{\sinh \pi(B-x) / D}{\cosh \pi(B-x) / D-\cos \pi z / D}\right] .
$$

The variations of $\left(\sigma_{z}-\sigma_{x}\right) / q$ down the centre line $\mathrm{C}-\mathrm{C}(x=0)$ and beneath the point 0 $(x=1 \cdot 1 B)$ for three typical layer depths $D / B=1,2$ and 3 , are shown in Fig. 24 . These 

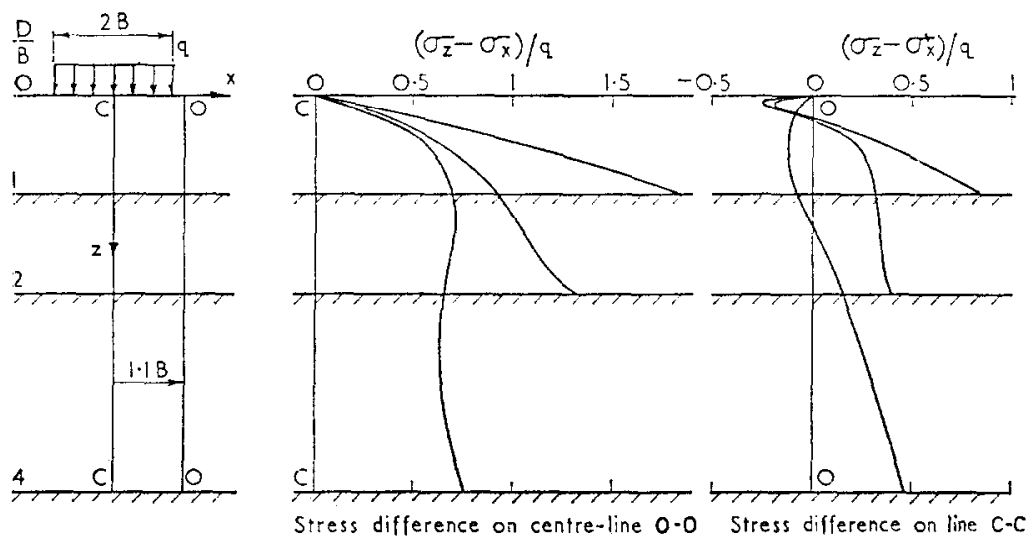

Fig. 24

distributions possess the surprising property that when used, in conjunction with equation (27), in equation (26) the surface settlement is found to be

$$
\begin{aligned}
S_{\mathrm{o}}(x) & =3 q / 2 \lambda \text { within the loaded area }(|x|<B) \\
& =0 \text { outside the loaded area }(|x|>B)
\end{aligned}
$$

It is known that when the base of the layer is rough the settlement becomes progressively less as the layer thickness decreases (Fig. 8) and this is what we expect. It is, therefore, to the smoothness of the base of this layer that we look for an explanation of the result (equation (29)). The shear stresses at a depth $D_{1}$ in the deeper layer (Fig. 23) will have the effect of restraining settlement compared with the smooth-based layer of depth $D_{1}$. However, the settlement is increased by the contribution of the material between this depth $D_{1}$ and the base $\left(z=D_{2}\right)$ of the layer. It turns out, apparently quite fortuitously, that these effects exactly compensate each other and, furthermore, that this balance is maintained irrespective of layer depth.

\section{REFERENCES}

Atkinson, J. H. (1973). The deformation of undisturbed London Clay. PhD thesis (Faculty of Engineering) University of London.

Awojobi, A. O. \& Gibson, R. E. (1973). Plane strain and axially symmetric problems of a linearly nonhomogeneous elastic half-space. Q. Jnl Mech. and Appl. Math. 26, 285-302.

Awojobi, A. O. (1974). The invariance of Gibson's law: for a layer on a smooth rigid base. Private communication; to be published.

Barden, L. (1963). Stresses and displacements in a cross-anisotropic soil. Géotechnique 13, No. 3, 198-210 (see also correspondence: 14, No. 3, 278; 15, No. 3, 317).

Bishop, A. W., Webb, D. L. \& Lewin, P. I. (1965). Undisturbed samples of London Clay from the Ashford Common Shaft: strength-effective stress relationships. Géotechnique 15, No. 1, 1-31.

Booker, J. R. \& Davis, E. H. (1972). A note on the plasticity solution to the stability of slopes in inhomogeneous clays. Géotechnique 22, No. 3, 509-513.

Borowicka, H. (1936). Influence of rigidity of a circular foundation slab on the distribution of pressures over the contact surface. Proc. Ist Int. Conf. Soil Mech., Cambridge, Mass. 1, 144.

Borowicka, H. (1943). Die Druckansbreitung im Halbraum bei linear zu nehmen dem Elastizitäts modul. Ing.-Archiv 14, 75.

Boussinesq, J. (1885). Applications des potentiels à l'étude de l'équilibre et du mouvement des solides élastiques. Paris: Gauthier-Villard.

Brown, P. T. (1969). Numerical analysis of uniformly loaded circular rafts on elastic layers of finite depth. Géotechnique 19, No. 2, 301.

Brown, P. T. (1972). The analysis of rafts on clay. PhD thesis, University of Sydney.

Brown, P. T. \& Gibson, R. E. (1972). Surface settlement of a deep elastic stratum whose modulus increases linearly with depth. Canad. Geot. Jnl 9, 467-476. 
Brown, P. T. \& Gibson, R. E. (1973). Rectangular loads on inhomogeneous elastic soil. Jnl Soil Mech. Fdns Div. Am. Soc. Civ. Engrs SM 10, 917-920.

Buckingham, E. (1921). Notes on the method of dimensions. Phil. Mag. 42.

Burland, J. B., Sills, G. C. \& Gibson, R. E. (1973). A field and theoretical study of the influence of nonhomogeneity on settlement. Proc. 8th Int. Conf. Soil Mech., Moscow 1.3, 39-46.

Carrier, W. D., III \& Christian, J. T. (1973). Rigid circular plate resting on a non-homogeneous elastic half-space. Géotechnique 23, No. 1, 67-84.

Crawford, C. B. \& Burn, K. N. (1962). Settlement studies on the Int. Sinai Hospital, Toronto. Eng. Jnl Can. Soc. Civ. Engrs 46 (5), 31-37.

Davis, E. H. \& Poulos, H. G. (1963). Triaxial testing and three-dimensional settlement analysis. Proc. 4th Australian-New Zealand Conf. Soil Mech., 233-243.

Davis, E. H. \& Poulos, H. G. (1968). The use of elastic theory for settlement prediction under threedimensional conditions. Géotechnique 18, No. 1, 67-91.

Davis, E. H. \& Booker, J. R. (1973). The effect of increasing strength with depth on the bearing capacity of clays. Géotechnique 23, No. 4, 551-563.

Drucker, D. C. \& Prager, W. (1952). Soil mechanics and plastic analysis or limit design. Q. Appl. Math. $10,157$.

Duncan, I. M. \& Seed, H. B. (1966). Anisotropy and stress orientation in clay. Proc. Am. Soc. Civ. Engrs 92, 81-104.

Ferrar, W. L. (1941). Algebra, 138-141. Oxford Univ. Press.

Fröhlich, O. K. (1934). Druckverteilung im Baugrunde. Vienna: Springer.

Gerrard, C. M. \& Harrison, W. J. (1970). Circular loads applied to a cross-anisotropic half-space. Tech. Paper No. 8, Geomech. Div. CSIRO, Australia.

Gerrard, C. M. \& Harrison, W. J. (1971). The analysis of a loaded half-space comprised of anisotropic layers. Tech. Paper No. 10, Geomech. Div. CSIRO, Australia.

Gibson, R. E. \& Morgenstern, N. R. (1962). A note on the stability of cuttings in normally consolidated clays. Géotechnique 12, No. 3, 212-216.

Gibson, R. E. (1967). Some results concerning displacements and stresses in a non-homogeneous elastic half-space. Géotechnique 17, No. 1, 58-67 (see also corrigenda: 18, No. 2, 275-276; 19, No. 1, 160-161).

Gibson, R. E., Brown, P. T. \& Andrews, K. R. F. (1971). Some results concerning displacements in a nonhomogeneous elastic layer. Zeit. f. ang. Math. Phys. 22, 855-864.

Gibson, R. E. \& Sills, G. C. (1971). Some results concerning the plane deformation of a non-homogeneous elastic half-space. Proc. Roscoe Mem. Symp., Cambridge, 564-572.

Gibson, R. E. \& Sills, G. C. (1974). Settlement of a strip load on a non-homogeneous orthotropic incompressible elastic half-space. Unpublished work.

Gibson, R. E. \& Kalsi, G. (1974). Some remarks on the surface settlement of a linearly inhomogenous orthotropic elastic half-space. Unpublished work.

Griffith, J. H. (1929). The pressures under substructures. Engng and Constn. 1, 113-119.

Hanna, T. H. (1965). Discussion: The elastic properties of a dense glacial till deposit. Can. Geot. J. 2, 129-131.

Hayashi, K. (1921). Theorie des Trägers auf elasticscher Unterlage. Berlin: Springer.

Hearmon, R. F. S. (1961). An introduction to applied anisotropic elasticity. Oxford Univ. Press.

Henkel, D. J. (1971). The relevance of laboratory measured parameters in field studies. Proc. Roscoe Mem. Symp. Cambridge, 669-675.

Hetényi, M. (1946). Beams on elastic foundations. Univ. Mich. Press.

Heyman, J. (1973a). Simple plasticity theory applied to soil mechanics. Proc. Symp. Plasticity in Soil Mech, Cambridge Eng. Dept, 161-172.

Heyman, J. (1973b). The stability of a vertical cut. Int. Jnl Mech. Sci. 15. 845-854.

Holl, D. L. (1940). Stress transmission in earths. Proc. Highway Res. Board 20.

Kérisel, J. \& Quatre, M. (1966). Tassements sous les fondations: méthode de prévision à partir de l'appareil triaxial. Ann. Ponts Chauss. 136, 143-164. (Translation in Civ. Eng. Publ. Wks Rev. 63, 531535; 661-666) (1968).

Klein, G. K. (1956). A study of non-homogeneity of discontinuities in deformations and of other mechanical properties of the soil in the design of structures on solid foundations. Sb. Trud. Mosk. inzh.-stroit. Inst. 14.

Koronev, B. G. (1957). A die resting on an elastic half-space, the modulus of elasticity of which is an exponential function of depth. Dokl. Akad. Nauk SSSR 112, 5 .

Lambe, T. W. (1964). Methods of estimating settlements. Jnl Soil Mech. Fdns Div. Am. Soc. Civ. Engrs 90, 43-67.

Lamé, G. \& Clapeyron, B. P. E. (1833). Mémoire sur l'équilibre intérieur des corps solides homogènes. Mém. par divers savants 4, (Paris). (Published $J n l f$. math. 7, (1831); report on paper by Poinsot \& Navier dated 1828.)

Lamé, G. (1852). Leçons sur la théorie mathématique de l'élasticité des corps solides. Paris. 
Lekhnitskii, S. G. (1962). Radial distribution of stresses in a wedge and in a half-plane with variable modulus of elasticity. Prinkl. Mat. Mekh. 26, 1.

Lekhnitskiī, S. G. (1963). Theory of elasticity of an anisotropic elastic body. San Francisco: Holden-Day.

Livneh, M. \& Greenstein, J. (1973). The bearing capacity of footings on non-homogeneous clays. Proc. 8th Int. Conf. Soil Mech., Moscow 1.3, 151-153.

Love, A. E. H. (1928). The stress produced in a semi-infinite solid by pressure on part of the boundary. Phil. Trans. Roy. Soc. (A) 228, 377-420.

Mayer, A. (1954). Joseph Boussinesq 1842-1929. Géotechnique 4, No. 1, 3-5.

Michell, J. H. (1900). The stress in an aeolotropic elastic solid with an infinite plane boundary. Proc. Lond. Math. Soc. 32, 247-258.

Mikhlin, S. G. (1935). A plane problem in elasticity theory for a non-homogeneous medium. Seismo. Inst. Acad. Sci. USSR 66.

Milovic, D. M. \& Tuzot, G. 1970. Bi-dimensional stress distribution in an anisotropic layer of finite thickness. Géotechnique 20, No. 2, 198-203.

Milovic, D. M. (1972). Stresses and displacements in an anisotropic layer due to a rigid circular foundation. Géotechnique 22, No. 1, 169-174.

Mitchell, R. J. (1972). Some deviations from isotropy in a lightly overconsolidated clay. Géotechnique 22, No. 3, 459-467.

Moore, P. J. \& Spencer, G. K. (1969). Settlement of building on deep compressible soil. Jnl Soil Mech. Fdns Div Am. Soc. Civ. Engrs 95, 769-790.

Nayak, M. (1960). Elastic settlement of a cross-anisotropic medium under axisymmetric loading. Soils and Foundations (Jap. Soc. Soil Mech. Fdn Engng) 13 (2), 83-90.

Newmark, N. M. (1942). Influence charts for computation of stresses in elastic foundations. Bull. No. 338, Univ. of Illinois, Eng. Expt Station.

Newmark, N. M. (1947). Influence charts for computation of vertical displacements in elastic foundations. Bull. No. 367, Univ. of Illinois, Eng. Expt Station.

Pérez-La Salvia, H., Luscher, U., Alvarez-Stelling, J. \& Gicot, O. (1966). Load-settlement-time behaviour of footings on clay. MIT Res. Report R66-27, Dept of Civ. Eng.

Pickering, D. J. (1970). Anisotropic elastic parameters for soil. Géotechnique 20, No. 3, 271-276.

Popov, G. Ya. (1959). Bending of an unbounded plate supported by an elastic half-space with a modulus of elasticity varying with depth. Prinkl. Mat. Mekh. 23, 1095.

Poulos, H. G. (1967). The use of the sector method for calculating stresses and displacements in an clastic mass. Proc. 5th Australian-New Zealand Conf. Soil Mech., Auckland, 198-204.

Poulos, H. G. \& Davis, E. H. (1974). Elastic solutions for soil and rock mechanics. New York: John Wiley.

Sherman, D. I. (1958). On the problem of plane strain in non-homogeneous media. Proc. Symp. on nonhomogeneity in elasticity and plasticity (Warsaw) 3. London: Pergamon Press.

Simons, N. S. (1971). The stress-path method of settlement applied to London Clay. Proc. Roscoe Mem. Symp., Cambridge, 241-252.

Skempton, A. W. \& Henkel, D. J. (1957). Tests on London Clay from deep borings at Paddington, Victoria and the South Bank. Proc. 4th Int. Conf. Soil Mech., London 1, 100-106.

Skempton, A. W. (1970). The consolidation of clays by gravitational compaction. Q. Jnl Geol. Soc. Lond. 125 (499), 373-408.

Steinbrenner, W. (1934). Tafeln zur Setzungsberechnung. Die Strasse 1, 121-4.

Terzaghi, K. (1955). Evaluation of coefficients of subgrade reaction. Géotechnique 5, No. 4, 297-326.

Ward, W. H., Samuels, S. G. \& Butler, M. E. (1959). Further studies of the properties of London Clay. Géotechnique 9, No. 2, 33-58.

Ward, W. H., Marsland, A. \& Samuels, S. G. (1965). Properties of the London Clay at the Ashford Common shaft. Géotechnique 15, No. 4, 321-344.

Winkler, E. (1867). Die Lehre von Elastizität und Festigkeit, 182. Prague.

Wroth, C. P. (1971). Some aspects of the elastic behaviour of overconsolidated clay. Proc. Roscoe Mem. Symp., Cambridge, 347-361.

Zaretsky, Y. K. \& Tsytovich, N. A. (1965). Considerations of heterogeneity and non-linear deformations of the base in the design of rigid foundations. Proc. 6th Int. Conf. Soil Mech., Montréal 2, 222-225.

\section{VOTE OF THANKS}

Dr Burland said that one of the attractions and challenges of geotechnics was the number and variety of disciplines and approaches it embraced. The thirteen previous Rankine Lectures had amply demonstrated this. Although the range of disciplines was wide, there was a central unifying feature-the practical nature of the subject; this was generated primarily by the engineer's need to understand and predict the behaviour of the ground. Progress in 
geotechnics depended on two vital ingredients: observation and analysis. Dr Burland said that there was often tension between the two, and it was right that there should be, but that the engineer ignored either at his peril.

He continued that in the past, the majority of Rankine Lectures had been concerned primarily with observations and measurements and their interpretation, and that those present had heard a brilliant and masterly lecture on the analytical method in soil mechanics delivered by one of its best known and most outstanding exponents. The subject was particularly timely in view of the current tendency towards increased sophistication in predictions using powerful numerical techniques and complex material laws. More than ever before there was a need for clear and precise analytical solutions to fundamental yet essentially practical problems. Such solutions provided the vital framework on which to hang experience and judgement.

Professor Gibson had shown us that the analytical method was much more than the ability to solve mathematical equations although Dr Burland received the impression that it was important to be able to do so. The fact that he had shown those present so few equations was a cause for relief and gratitude on their part and for congratulation on that of Professor Gibson. The method appeared to consist much more in asking the right questions and formulating the right analytical models to answer those questions. Dr Burland said that he also suspected that there was a touch of mysticism about the method; to very few people was granted the ability to get the oracle to talk: Professor Gibson was one of the few with this gift, and perhaps the oracle did not always talk for him either.

Dr Burland went on to mention a number of qualities that characterized Professor Gibson's. work. Elegance was one of them and flare another. Much of his work also contained an element of surprise, an element of the unexpected; as had been seen that evening his work often yielded results which were contrary to one's intuition yet obvious once they had been demonstrated. This had occurred sufficiently frequently for the engineer to be very wary about trusting to his own judgement too much.

Dr Burland felt sure that those present would agree with him that the Fourteenth Rankine Lecture represented an important milestone in the progress of soil mechanics. The Paper would be thoughtfully studied and widely used for many years to come.

It was both a great honour and a very great pleasure for Dr Burland to propose a sincere vote of thanks to Professor Gibson for delivering the Lecture.

The vote of thanks was accorded with acclamation 